\title{
Industrial biochar systems for atmospheric carbon removal: a review
}

\author{
Samer Fawzy ${ }^{1} \cdot$ Ahmed I. Osman $^{1}$ (D) Haiping Yang ${ }^{2} \cdot$ John Doran $^{3} \cdot$ David W. Rooney $^{1}$
}

Received: 4 February 2021 / Accepted: 16 February 2021 / Published online: 11 March 2021

(c) The Author(s) 2021

\begin{abstract}
In the context of climate change, there is an urgent need for rapid and efficient methods to capture and sequester carbon from the atmosphere. For instance, production, use and storage of biochar are highly carbon negative, resulting in an estimated sequestration of $0.3-2 \mathrm{Gt} \mathrm{CO}_{2}$ year $^{-1}$ by 2050 . Yet, biochar production requires more knowledge on feedstocks, thermochemical conversion and end applications. Herein, we review the design and development of biochar systems, and we investigate the carbon removal industry. Carbon removal efforts are currently promoted via the voluntary market. The major commercialized technologies for offering atmospheric carbon removal are forestation, direct air carbon capture utilization and storage, soil carbon sequestration, wooden building elements and biochar, with corresponding fees ranging from 10 to 895 GBP (British pounds) per ton $\mathrm{CO}_{2}$. Biochar fees range from 52 to $131 \mathrm{GBP}$ per ton $\mathrm{CO}_{2}$, which indicates that biochar production is a realistic strategy that can be deployed at large scale. Carbon removal services via biochar are currently offered through robust marketplaces that require extensive certification, verification and monitoring, which adds an element of credibility and authenticity. Biochar eligibility is highly dependent on the type of feedstock utilized and processing conditions employed. Process optimization is imperative to produce an end product that meets application-specific requirements, environmental regulations and achieve ultimate stability for carbon sequestration purposes.
\end{abstract}

Keywords Biochar · Carbon removal · Climate change mitigation · Carbon stability · Carbon reservoirs

\begin{tabular}{|c|c|c|c|}
\hline \multicolumn{2}{|c|}{ Abbreviations } & $\mathrm{g} \mathrm{g}^{-1}$ & Gram per gram \\
\hline \multirow[t]{2}{*}{ AKTS } & Advanced kinetics and technology & GC-MS & Gas chromatography-mass spectrometry \\
\hline & solutions & $\mathrm{Gt} \mathrm{CO}_{2}$ year $^{-1}$ & Gigaton of carbon dioxide per year \\
\hline $\mathrm{Ca}^{2+}$ & Calcium ion & $\mathrm{H} / \mathrm{C}_{\text {org }}$ & Hydrogen to organic carbon molar ratio \\
\hline $\mathrm{CH}_{4}$ & Methane & $\mathrm{H}^{+}$ & Hydrogen ion \\
\hline $\mathrm{CO}_{2}$ & Carbon dioxide & $\mathrm{H}_{2} \mathrm{O}_{2}$ & Hydrogen peroxide \\
\hline EDXS & Energy-dispersive X-ray spectroscopy & HRTEM & High-resolution transmission electron \\
\hline FTIR & Fourier transform infrared spectroscopy & & microscopy \\
\hline \multicolumn{2}{|l|}{$\mathrm{g} \mathrm{cm}^{-3}$} & ICP-AES & $\begin{array}{l}\text { Inductively coupled plasma atomic emis- } \\
\text { sion spectrometry }\end{array}$ \\
\hline & & ICP-MS & Inductively coupled plasma mass \\
\hline \multirow{2}{*}{\multicolumn{2}{|c|}{$\begin{array}{l}\text { Disclaimer The views and opinions expressed in this paper do } \\
\text { not necessarily reflect those of the European Commission or the } \\
\text { Special EU Programmes Body (SEUPB). }\end{array}$}} & & spectrometry \\
\hline & & ICP-OES & $\begin{array}{l}\text { Inductively coupled plasma optical emis- } \\
\text { sion spectrometry }\end{array}$ \\
\hline \multicolumn{2}{|c|}{$\begin{array}{l}\triangle \text { Ahmed I. Osman } \\
\text { aosmanahmed01@qub.ac.uk }\end{array}$} & ICTAC & $\begin{array}{l}\text { International confederation for thermal } \\
\text { analysis and calorimetry }\end{array}$ \\
\hline \multirow{3}{*}{$\begin{array}{l}\text { Sc } \\
\text { Un } \\
\mathrm{Be}\end{array}$} & & $\mathrm{K}^{+}$ & Potassium ion \\
\hline & $\begin{array}{l}\text { mistry and Chemical Engineering, Queen's } \\
\text { lfast, David Keir Building, Stranmillis Road, }\end{array}$ & $\mathrm{K}_{2} \mathrm{Cr}_{2} \mathrm{O}_{7}$ & Potassium dichromate \\
\hline & AG, Northern Ireland, UK & $\mathrm{KMnO}_{4}$ & Permanganate \\
\hline \multirow{3}{*}{$\begin{array}{l}\mathrm{St} \\
\mathrm{Ur} \\
\mathrm{Cl}\end{array}$} & oratory of Coal Combustion, Huazhong & $\mathrm{m}^{2} \mathrm{~g}^{-1}$ & Square meter per gram \\
\hline & Science and Technology, Wuhan 430074 , & $\mathrm{Mg}^{2+}$ & Magnesium ion \\
\hline & & Mpa & Megapascal pressure unit \\
\hline The I & ntre at Letterkenny Institute of Technology, & $\mathrm{N}_{2} \mathrm{O}$ & Nitrous oxide \\
\hline
\end{tabular}




\begin{tabular}{|c|c|}
\hline $\mathrm{Na}^{+}$ & Sodium-ion \\
\hline NEXAFS & $\begin{array}{l}\text { Near-edge X-ray absorption fine structure } \\
\text { spectroscopy }\end{array}$ \\
\hline$n g L^{-1}$ & Nanogram per litre \\
\hline $\mathrm{NH}_{4}^{+}$ & Ammonium ion \\
\hline $\mathrm{nm}$ & Nanometre \\
\hline $\mathrm{O} / \mathrm{C}_{\text {org }}$ & Oxygen to organic carbon molar ratio \\
\hline${ }^{\circ} \mathrm{C} \min ^{-1}$ & Degrees Celsius per minute \\
\hline Py-GC-MS & $\begin{array}{l}\text { Pyrolysis-gas chromatography-mass } \\
\text { spectrometry }\end{array}$ \\
\hline REDD + & $\begin{array}{l}\text { Reducing emissions from deforestation } \\
\text { and forest degradation }\end{array}$ \\
\hline UNFCCC & $\begin{array}{l}\text { United Nations Framework Convention on } \\
\text { Climate Change }\end{array}$ \\
\hline$\mu \mathrm{g} \mathrm{g}^{-1}$ & Microgram per gram \\
\hline
\end{tabular}

\section{Introduction}

Acknowledgement of the realities associated with the shift in climate patterns started in 1979. However, actual global efforts to combat the detrimental effects of increased greenhouse gas concentrations took place in the early 90 s by introducing and adopting the United Nations Framework Convention on Climate Change (UNFCCC). Through the UNFCCC, the Kyoto protocol was introduced in 1997 and then adopted in 2005, which introduced emission reduction commitments and provided various mechanisms to achieve such targets. In 2015, the Paris agreement was adopted which laid out further commitments, objectives, enhanced monitoring and compliance regulations and introduced further support mechanisms. The main objective of the agreement was to limit global temperature increase to $2{ }^{\circ} \mathrm{C}$ by the end of the century and pursue efforts to cap it at $1.5^{\circ} \mathrm{C}$. With the current emission rates, we will likely reach the $1.5^{\circ} \mathrm{C}$ within the next couple of decades, and if emissions continue to increase an estimated $3-4{ }^{\circ} \mathrm{C}$ will be realized by the end of the century. There is evidence showing that the current emission reduction efforts, in addition to the future emission commitments declared, are not sufficient to achieve the targets stipulated by the Paris agreement (Fawzy et al. 2020).

Fawzy et al. discussed three main strategies for mitigation of climate change, namely conventional mitigation, negative emissions and radiative forcing geoengineering techniques. Conventional mitigation focuses on emission reduction through renewable energy technologies, efficiency gains, fuel switching, nuclear power and carbon capture and storage technologies. Negative emissions technologies also referred to as carbon removal methods are a new set of methods that include a number of biogenic, technological and hybrid carbon sequestration techniques. This includes bioenergy carbon capture and storage, biochar, soil carbon sequestration, forestation, wetland restoration and construction, direct air carbon capture and storage, enhanced terrestrial weathering, ocean fertilization and alkalinity enhancement, biomass utilization in the built environment as well as mineral carbonation. On the other hand, radiative forcing geoengineering techniques alter the earth's energy budget to reduce and stabilize global temperatures. However, such techniques are still at a conceptualization stage and do not solve the root cause of the problem, that is the high concentration levels of greenhouse gases in the atmosphere. In reality, the world can mainly rely on the first two strategies, (1) reducing emissions, and (2) removing atmospheric carbon, to reduce global temperatures (Fawzy et al. 2020).

Based on the current state of climate emergency, the development of effective and financially viable carbon removal projects is paramount. Biochar has been recognized as a very promising negative emissions technology that promotes atmospheric carbon capture and integrated utilization and storage (Osman et al. 2020a). Atmospheric carbon removal is achieved through the photosynthetic process that takes place during plant growth coupled with a consequent thermochemical conversion process that results in a solid carbonaceous material, referred to as biochar, that carries a very stable form of carbon which can resist thermal and biological degradation for extended periods, in the form of centuries to millennia. The biochar can then be safely stored in soils, building structures and various carbon sinks, where additional services can be extracted depending on the final application. It is estimated that by 2050 , carbon removal via biochar will be in the range of $0.3-2 \mathrm{Gt} \mathrm{CO}_{2}$ year $^{-1}$ (Fawzy et al. 2020). This literature review explores various areas related to biochar production as well as the current status of the carbon removal industry and provides insight that can guide in the design and development of an industrial biochar production system geared towards atmospheric carbon removal. The investigation will cover potential feedstocks, common feedstock analysis techniques, production technologies, biochar properties and characterization techniques, the impact of processing parameters on biochar properties and yield, by-product valorization, and introduces potential applications and carbon reservoirs. Furthermore, the article will present the current status of the emerging carbon removal market and discuss market participation requirements.

\section{Feedstocks}

\section{Potential feedstocks}

Lignocellulosic biomass includes agricultural and forestry residues, agro-industrial wastes, short rotation forestry and dedicated energy crops, which constitutes a mixture of natural polymers, namely cellulose, hemicellulose and lignin. 
Lignocellulosic biomass is renewable and abundantly available, deeming it a desirable feedstock for biochar production (Yaashikaa et al. 2019). In addition to lignocellulosic materials, nutrient-rich non-lignocellulosic materials can also be utilized, such as livestock manure, sewage sludge and algae (Li and Jiang 2017). Table 1 presents various examples of the potential feedstocks discussed. Feedstock characteristics, availability, cost and intended biochar application are important aspects to consider when selecting an appropriate feedstock material. Furthermore, for biochar certification purposes, feedstock eligibility needs to be taken into account (EBC 2012; IBI 2015).

Although the use of residual biomass supports the circular economy concept, some of the major concerns related to such feedstock material include seasonal availability, quality attribute variability and price volatility due to competition for other uses. Moreover, logistics is another major challenging area (Fawzy et al. 2020; Kenney et al. 2013). To overcome such challenges, it is recommended that feedstocks are secured through professional waste management companies that can enter into long-term contractual supply agreements. Agro-industrial wastes are more stable in terms of logistics, availability and price volatility since large volumes can be secured from single point sources under long-term contractual agreements.

The most robust feedstock supply system would be based on the cultivation of fast-growing dedicated crops or shortrotation plantations grown on marginal land, where full control can be exercised, achieving supply and cost stability as well as control over material characteristics and quality. Moreover, a dedicated carbon cropping system adds another dimension to carbon removal, in which further sequestration can be realized via soil organic carbon throughout the plantation's lifecycle. Dedicated cultivation in some cases can lead to detrimental effects in relation to greenhouse gas emissions, through land-use change, if carbon-dense ecosystems are converted to dedicated plantations. Furthermore, competition with food production for resources, such as land, nutrients and water, is also identified as a negative aspect related to dedicated biomass cultivation. It is imperative that dedicated cultivation is carried out sustainably on marginal lands, in addition to using resources that do not add any stress or competition with food production systems (Fawzy et al. 2020).

\section{Feedstock analysis techniques}

Biomass characteristics play a very important role in defining the material's behaviour within the conversion process, as well as the final biochar quality and thus require appropriate analysis to better understand its physicochemical attributes. This section will present the major feedstock characterization techniques reported in the literature.

\section{Proximate, ultimate and heating value analyses}

Proximate analysis is the most common analytical technique utilized for biomass characterization. It includes the identification of various fractions (1) volatile matter, the vapour resulting from biomass heating (2) fixed carbon, the char fraction that remains after de-volatilization (3) moisture and (4) ash content, which is the inorganic fraction remaining after complete oxidation of biomass (Okafor and Daramola 2020; Basu 2013; Dhyani and Bhaskar 2018). Various methods are used to determine each of the fractions, as shown in Table 2, while fixed carbon is usually calculated by difference.

Ultimate analysis is carried out using an elemental analyser which involves the determination and quantification, in weight percentage, of carbon, hydrogen, nitrogen, sulphur and oxygen within biomass material. The results obtained

Table 2 Proximate analysis testing standards

\begin{tabular}{lll}
\hline & ASTM Standard & $\begin{array}{l}\text { British and European } \\
\text { Standard }\end{array}$ \\
\hline Moisture content & E1756-08 & BS EN 14775-3 \\
Volatile matter content & E872-82 & BS EN 15148:2009 \\
Ash content & E1755-01 & BS EN 14775:2009 \\
\hline
\end{tabular}

Table 1 Potential feedstock examples

\begin{tabular}{lll}
\hline Residual biomass & Agriculture & Rice straw, wheat straw, cotton stalks, corn stalks, fruit tree trimmings, tobacco stems, tea stems \\
Livestock & Cattle manure, chicken litter \\
Agro-industrial & Bagasse, empty fruit bunch, coconut shells, rice husk, corn cobs, olive cake, palm kernel shells \\
Municipal & Sewage sludge, municipal tree prunings \\
& Forestry & Logs, chips and bark from tree thinning activities \\
& Industrial & Sawdust, slab timber, sawmill wood chips and offcuts, distillers' grains \\
Dedicated Biomass & Herbaceous & Miscanthus, Arundo donax, switchgrass, bamboo, elephant grass \\
& Woody & Short rotation coppice, e.g. poplar, willow, eucalyptus, casuarina, sesbania \\
& Aquatic & Microalgae and macroalgae
\end{tabular}


are quite useful as they describe and quantify the major elemental constituents. Various ratios derived from such results provide an opportunity for comparison between feedstocks. The results obtained through an ultimate analysis can also be utilized to compute biomass heating values (Okafor and Daramola 2020; Basu 2013; Dhyani and Bhaskar 2018).

Calorific or heating value of biomass is the heat released under combustion. The higher heating value is usually obtained using a bomb calorimeter using various test methods, e.g. ASTM D5865-13. The heating value can also be computed using the results obtained from the ultimate analysis. Huang and Lo recently developed a highly accurate empirical correlation for computing the higher heating value using elemental results, as shown in Eq. 1 (Huang and Lo 2020). Consequently, the lower heating value can be computed using Eq. 2 (Osman et al. 2018), which excludes moisture present in the biomass and water vapour formed during the combustion process (Acar and Ayanoglu 2012). Although biochar for atmospheric carbon removal does not incorporate combustion at any stage, information relating to heating value is required when constructing process energy balances and calculating process efficiencies. biomass is occupying, including the space pores within the biomass particles, and is usually expressed in $\mathrm{kg} \mathrm{m}^{-3}$ (Cai et al. 2017). Measurement of bulk density can be carried out according to various standards such as ASTM E873-82 and BS EN ISO 17828:2015.

Furthermore, microstructural characterization can be carried out using the Brunauer-Emmett-Teller nitrogen physisorption method, in which specific surface area, pore structure and pore volume are measured. Surface area and porosity are very important attributes concerning the final biochar product and are usually measured pre-and post-conversion. The type of feedstock and production parameters define such structural characteristics. Furthermore, scanning electron microscopy is a qualitative analytical imaging technique used for investigating surface morphology (Osman et al. 2019; Mulabagal et al. 2017).

\section{Chemical structure and functional group analyses}

$\mathrm{X}$-ray powdered diffraction is an analytical technique that measures material crystallinity. The degree of crystallinity is useful in describing the material's structural properties.

Higher heating value $=0.3443 *$ carbon $\%+1.192 *$ hydrogen $\%-0.113 *$ oxygen $\%$

$$
-0.024 * \text { nitrogen } \%+0.093 * \text { sulphur } \%
$$

Lower heating value $=$ Higher heating value $-0.212 *$ hydrogen $\%$ $-0.0245 *$ moisture $\%-0.008 *$ oxygen $\%$

\section{Physical property analysis}

Physical properties such as particle size and bulk density are important as such properties directly impact logistical and operational efficiencies. Various analytical methods can be utilized to further understand biomass physical attributes. Sieving or image particle analysis is usually carried out to determine particle size distribution. Sieving analysis can follow various standards such as ANSI S424.1 and BS EN ISO 17827-1:2016. Image analysis can reveal more information about particle size, shape and dimensions (Cai et al. 2017). Particle size is an important aspect as it directly impacts heat transfer efficiencies during thermochemical conversion. Bulk density is another important physical property that has a profound effect on logistical efficiencies and production throughput. Most thermochemical conversion technologies are limited by volumetric capacity; therefore, bulk density plays a crucial role in defining how much material can be processed within a specific time frame. Bulk density is a function of particle size, particle density, moisture content, and particle shape. Bulk density is the ratio of the weight of biomass particles to the total volume the
Furthermore, this analytical tool can indicate the presence of various mineral phases. X-ray powdered diffraction analysis is recommended pre- and post-conversion to observe phase and structural changes, which may influence the material's physical behaviour. The degree of crystallinity can be computed using the crystallinity index developed by Segal, as shown in Eq. 3. Various methods are available to quantify the degree of crystallinity; however, Segal's index is a common approach used within the literature (Ferrer et al. 2016).

$\% \mathrm{CRI}=\frac{\left(I_{002}-I_{\mathrm{am}}\right)}{I_{002}} \times 100$

where $I_{002}$ is the maximum intensity point of the crystalline peak at $2 \theta=22.6^{\circ}$, and $I_{\text {am }}$ is the minimum intensity point of the amorphous peak at $2 \theta=18.05^{\circ}$.

Energy-dispersive X-ray spectroscopy (EDXS) is a common analytical tool usually deployed to investigate surface chemical structure. This analysis is based on the top $10 \mathrm{~nm}$ of the material surface. The surface elemental composition can be quantitatively identified, and elemental mapping can provide more information on particle distribution (Osman et al. 2019).

Fourier transform infrared spectroscopy (FTIR) is a qualitative technique usually deployed to detect functional groups within a biomass sample, indicating structural characteristics (Xu et al. 2013). FTIR analysis is recommended pre- and 
post-conversion to observe functional group evolution to understand the impact of changes related to compound physical and chemical properties that define functional capabilities and behaviour. Quantitative composition analysis can be carried out using FTIR coupled with statistical modelling, such as principal component analysis or partial least squares, to determine lignocellulosic fractions. This is referred to as chemometric analysis (Okafor and Daramola 2020; Ferrer et al. 2016; Xu et al. 2013). Lignocellulosic fractions can also be obtained via wet chemical analytical methods. Through the determination of neutral detergent fibre, acid detergent fibre and acid detergent lignin, the three lignocellulosic fractions, cellulose, hemicellulose and lignin, can be obtained (Dhyani and Bhaskar 2018).

Furthermore, biomass contains organic as well as inorganic compounds. Inorganic elements are present in the ash fraction and may consist of various species such as potassium, magnesium, iron, silicon, calcium, chlorine, phosphorus and sodium. The inorganic element content is usually a function of the specific type of biomass, soil characteristics and cultivation practices. Furthermore, heavy metals such as cadmium, mercury and lead may be present, which are usually classified as toxic elements. Inductively coupled plasma mass spectrometry (ICP-MS), inductively coupled plasma atomic emission spectrometry (ICP-AES) and inductively coupled plasma optical emission spectrometry (ICP-OES) are common analytical techniques for inorganic metal analysis (Okafor and Daramola 2020; Dhyani and Bhaskar 2018). EDXS analysis can also be used to detect surface inorganic element composition.

\section{Thermo-kinetic analysis}

Knowledge of a material's thermo-kinetic behaviour under various thermochemical conversion conditions is of great importance for the successful design and optimization of biochar production systems. The analysis comprises a thermal degradation investigation followed by kinetic modelling to identify the kinetic triplet, activation energy $\left(E_{\mathrm{a}}\right)$, reaction rate and pre-exponential factor $\left(k_{\mathrm{o}}\right)$. This information is very useful for the prediction of feedstock reaction progress under various thermal conditions. A thermal investigation is usually carried out using non-isothermal thermogravimetric analysis or differential scanning calorimetry. Furthermore, using the results obtained via thermogravimetric analysis or differential scanning calorimetry, various methods are applied to identify the kinetic parameters, namely modelfitting and model-free methods (Osman et al. 2020b, c; Akor et al. 2021). Model fitting techniques are, in general, not recommended by the international confederation for thermal analysis and calorimetry (ICTAC) kinetics committee due to the uncertainty related to the determination of kinetic parameters (Vyazovkin et al. 2011). The most common model-free methods reported in the literature include the single-step reaction method ASTM E-698, integral iso-conversional methods such as Flynn-Wall-Ozawa, and Kissinger-Akahira-Sunose and Friedman's differential iso-conversional method (Osman et al. 2020b, c; Akor et al. 2021; Mishra and Mohanty 2018; Luo et al. 2020; Bonilla et al. 2019). In general, all methods carry various drawbacks and may lead to errors and inaccuracies in computing kinetic parameters if mishandled (Vyazovkin et al. 2011; Luo et al. 2020). Various kinetic modelling software can be utilized for accurate analysis, such as advanced kinetics and technology solutions (AKTS) (Osman et al. 2020b, c; Akor et al. 2021), or kinetics neo software. In addition to computing the kinetic triplet, the modelling software can provide robust reaction predictions which is a powerful tool. Predictions cover several thermal profiles and modes, such as isothermal, nonisothermal and stepwise modes. The kinetic information can also be utilized further in chemical process simulation software for process output predictions and optimization.

\section{Biochar production technologies}

Various thermochemical conversion technologies can be utilized to produce biochar, namely pyrolysis, gasification, hydrothermal carbonization and flash carbonization (Kumar et al. 2020; Novotny et al. 2015). This section will discuss each of the conversion routes reported in the literature.

\section{Pyrolysis}

Pyrolysis is a thermal process that involves the degradation of biomass in an oxygen-free environment at temperatures between 300 and $900{ }^{\circ} \mathrm{C}$. Pyrolysis of biomass generally yields three distinct products: a solid fraction referred to as biochar, a liquid fraction and a gaseous fraction. The mechanism behind the pyrolytic degradation of lignocellulosic material is complex and has been discussed extensively in the literature (Uddin et al. 2018; Demirbas 2009). In general, many reactions take place in parallel and series. This includes dehydration, depolymerization, volatilization, charring, aromatization, decarboxylation, cracking, repolymerization and condensation (Kan et al. 2016). The literature presents a general consensus that biomass pyrolysis follows three main stages: (1) initial dehydration, (2) primary decomposition, followed by (3) secondary reactions. Thermal degradation of biomass usually occurs during the primary decomposition stage at $200-400{ }^{\circ} \mathrm{C}$, forming solid char. Primary decomposition is perhaps responsible for the majority of degradation taking place. This is followed by secondary reactions as the temperature increases, promoting further volatilization. 
Degradation pathways of lignocellulosic components have been extensively investigated in the literature. Hemicellulose decomposition occurs between 250 and $350{ }^{\circ} \mathrm{C}$, followed by cellulose, which decomposes between 325 and $400{ }^{\circ} \mathrm{C}$. On the other hand, lignin is more stable and usually decomposes at a temperature range between 300 and $550{ }^{\circ} \mathrm{C}$ (Kan et al. 2016). Osman et al. presented a detailed ten-step mechanism associated with the pyrolytic conversion of lignocellulosic biomass. The process presented primary and secondary reactions, where independent reactions related to lignin, cellulose and hemicellulose occur simultaneously. Despite each of these components' independent behaviour, char, vapours and tar are produced (Osman et al. 2020b). Figure 1 provides a depiction of the general pyrolysis mechanism of lignocellulosic material.

End product yield distribution and properties are a function of feedstock characteristics and process parameters, such as temperature, heating rate, residence time, particle size and type of reactor used. The pyrolytic process can be categorized into various types, namely slow, intermediate, fast and flash pyrolysis, where the classification is mainly based on the rate of heat transfer. Slow pyrolysis is a mature and robust technology for the production of char. It is characterized by a slow heating rate, with a typical processing temperature ranging $400-600{ }^{\circ} \mathrm{C}$, although temperatures lower and higher than this range are also reported in the literature, and a residence time ranging from hours to days. Slow pyrolysis is known to achieve a high char yield, and the literature reports a range between $\sim 20$ and $50 \%$, which mainly depends on the feedstock and processing parameters (Kumar et al. 2020; Tripathi et al. 2016; Wang et al. 2020; Li et al. 2020; Tisserant and Cherubini 2019). Slow pyrolysis is usually carried out in batch process fixed bed reactors, retorts or converters (Brassard et al. 2017; Hornung 2012).

Intermediate pyrolysis is carried out at a similar processing temperature range, however, at slow to moderate heating rates and provides good char yields $\sim 20$ and $40 \%$. Furthermore, production is continuous and usually takes up to $30 \mathrm{~min}$. Typical reactors include externally and internally heated rotary kilns, as well as auger-based kilns (Brassard et al. 2017; Hornung 2012; Waluyo et al. 2018; Yang et al. 2014). Many commercial models available in the market are based on these reactor designs. The literature sometimes classifies intermediate pyrolysis under slow pyrolysis.

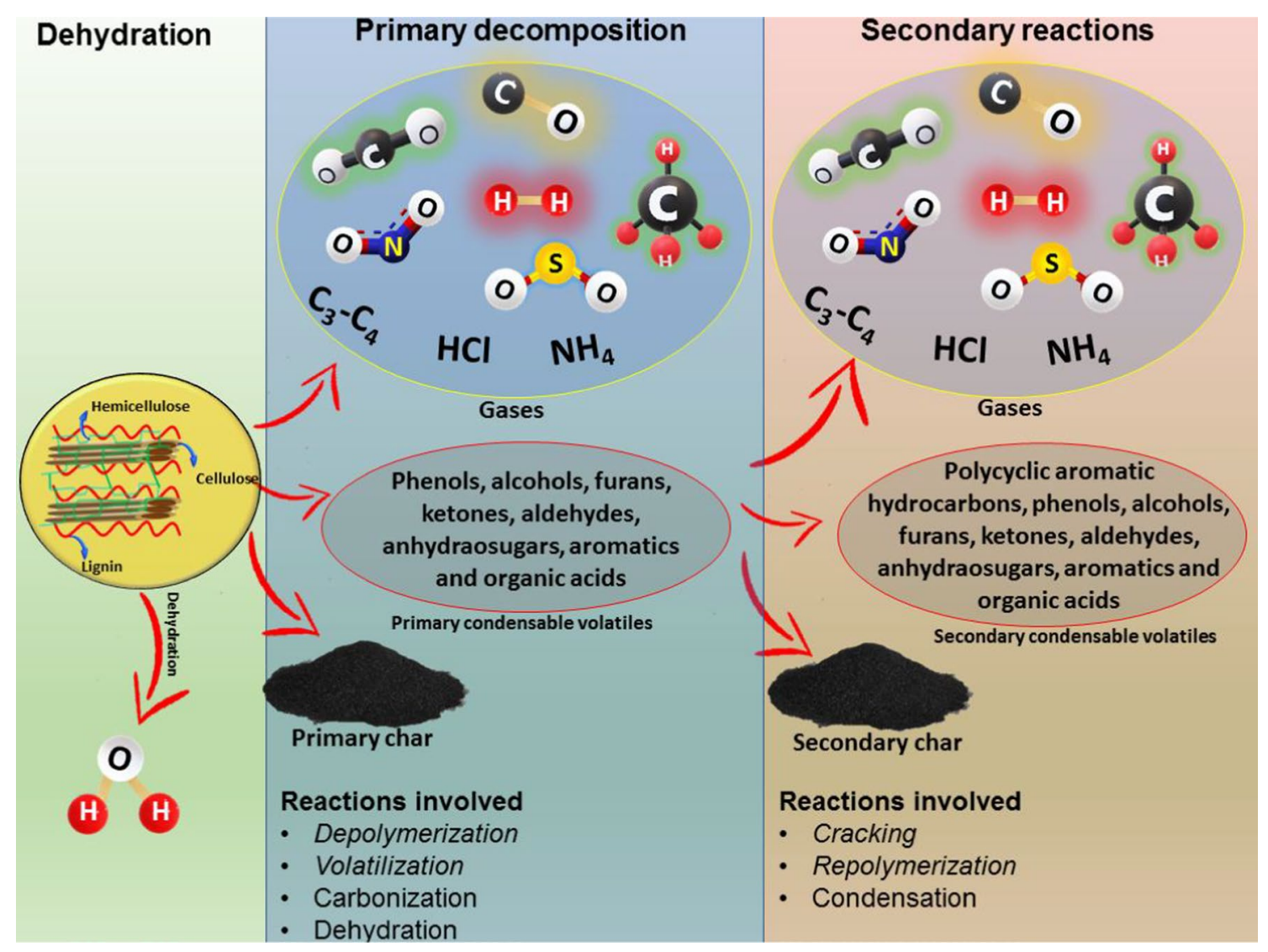

Fig. 1 Pyrolysis mechanism of lignocellulosic material, showing reactions involved and the formation of primary char, gases and primary condensable volatiles during the primary decomposition stage.
Due to solid-vapour interactions, secondary reactions such as cracking, repolymerization and condensation allow for secondary char, gases and secondary condensable volatiles to be produced 
Fast and flash pyrolysis, while they operate at a similar temperature range, the heating rate is much faster and residence time is usually in the range of seconds, favouring biooil production and offers a typical biochar yield of 5-20\% (Kumar et al. 2020; Tripathi et al. 2016; Wang et al. 2020; Li et al. 2020; Tisserant and Cherubini 2019). Typical reactors for fast and flash pyrolysis include bubbling fluidized bed reactors, circulating fluidized bed reactors. Other designs include ablative and cone reactors, as well as the twin screw reactor which is based on a mechanical fluidized bed design (Brassard et al. 2017; Hornung 2012). Furthermore, various pyrolysis forms are also reported in the literature, which include microwave-assisted pyrolysis, vacuum pyrolysis and hydropyrolysis (Tripathi et al. 2016). For efficient biochar production, reactors that are based on slow and intermediate pyrolysis are the most suited. Continuous rotary kilns, as well as auger-based kilns, are reliable and mature technologies. Various reactor manufacturers offer features and configurations that are unique to their designs. Not all reactors are produced equally.

\section{Gasification}

Gasification is another thermochemical conversion that produces biochar as a by-product. Biomass gasification is usually carried out at a temperature range of $700-1000{ }^{\circ} \mathrm{C}$, in a partially oxidized environment, using air, steam or oxygen. While the main products of gasification are similar to pyrolysis, this process favours the production of syngas. The literature reports a typical yield of $\sim 5,10$ and $85 \%$ for char, oil and syngas, respectively (Kumar et al. 2020; Wang et al. 2020; Tisserant and Cherubini 2019). The low char yield achieved via this route deems this technology inefficient for the production of biochar. Gasification is more suited for energy production and the production of various chemicals that are synthesized from syngas.

\section{Hydrothermal carbonization}

Hydrothermal carbonization is a thermochemical technology that carries out biomass conversion in an aqueous inert environment under high pressure, with residence time being in the form of hours to days (Afolabi et al. 2020; Malghani et al. 2013; Sharma et al. 2019). Generally, hydrothermal carbonization can be categorized into low temperature, carried out below $300{ }^{\circ} \mathrm{C}$ and high temperature, carried out in the range of $300-800{ }^{\circ} \mathrm{C}$. Hydrothermal carbonization offers high conversion yields in relation to char with $\sim 65 \%$ and $30-60 \%$ for low- and high-temperature hydrothermal carbonization, respectively (Kumar et al. 2020). The process is very suited for processing wet biomass since there is no requirement for pre-drying of the feedstock. Although this process offers high biochar yields, which is comparable to slow pyrolysis, the biochar physicochemical properties may be different compared to those produced by slow pyrolysis. Malghani et al. produced biochar using slow pyrolysis and hydrothermal carbonization using the same feedstock, corn silage; however, the resulting biochar carried different chemical properties, physical appearance and decomposition behaviour when applied to soil. The researchers concluded that hydrothermal carbonization biochar decomposed more rapidly in all types of soils tested, compared to those produced via slow pyrolysis and discussed its potential to stimulate other greenhouse gas emissions such as $\mathrm{CH}_{4}$, and $\mathrm{CO}_{2}$ resulting from the priming of organic matter. On the other hand, slow pyrolysis biochar exhibited carbon stability and is deemed more suitable for climate change mitigation (Malghani et al. 2013). According to the European biochar certificate, chars produced via hydrothermal carbonization are not classified as biochar (EBC 2012). Hydrothermal carbonization may be more suitable for producing biocarbon for energy production, since the chars produced possess superior characteristics in terms of low ash content and high calorific value (Afolabi et al. 2020).

\section{Flash carbonization}

Flash carbonization is another thermochemical process that requires the ignition and control of a flash fire at an elevated pressure within a packed biomass bed. The mechanism involves fire moving upwards, while a flow of air moves downwards which triggers the conversion of lignocellulosic material mainly into gaseous and solid fractions. This process usually requires a residence time under $30 \mathrm{~min}$, while a temperature between 330 and $650{ }^{\circ} \mathrm{C}$ is maintained (Kumar et al. 2020; Nartey and Zhao 2014; Antal et al. 2003; Hirst et al. 2018; Nunoura et al. 2006). The literature reports that biochar yields via flash carbonization ( 28 and 32\%) are inline with those produced by slow and intermediate pyrolysis, while some manufacturers claim higher biochar yields. The main disadvantage associated, however, is the requirement for elevated pressure (Kumar et al. 2020; Li et al. 2020). Flash carbonization is another potential technology for the efficient production of biochar. This technology is currently employed by commercial biochar producers.

\section{Biochar properties and characterization techniques}

The decisive factor associated with the ability of biochar to mitigate climate change is its carbon stability. Furthermore, it is important to note that biochar needs to be effectively applied to potential carbon reservoirs for long-term storage to qualify as a carbon removal strategy. Therefore, it is imperative to understand the various properties associated 
with biochar to effectively carry out optimization for application-specific requirements while maintaining long-term carbon stability. This section will further explore biochar's chemical properties and composition, physical properties, hydrological properties as well as discuss properties that are related to carbon stability and carbon stability assessment techniques.

\section{Chemical properties and composition}

In general, chemical properties and composition of biochar represent the chemical characteristics of the original feedstock and the extent of thermal processing undertaken. The literature reports various chemical properties for biochar, namely $\mathrm{pH}$, surface charge, cation exchange capacity, electrical conductivity, volatile matter, fixed carbon and ash content, elemental composition, heavy metals and polycyclic aromatic hydrocarbons (Yargicoglu et al. 2015; Qambrani et al. 2017; Chen et al. 2019; Ra et al. 2016). Biochar is mainly composed of fixed carbon, volatile matter, moisture and ash. Unlike carbon in original biomass feedstocks, the carbon structure within biochar is mainly aromatic, which includes both amorphous and crystalline phases; however, non-aromatic carbon structures also exist within the biochar (Qambrani et al. 2017). The elemental composition of biochar generally includes carbon, hydrogen, nitrogen, sulphur and oxygen as well as inorganic minerals such as potassium, phosphorus, calcium, magnesium, iron, silicon and sodium, which exist within the ash fraction. While carbon content constitutes the major fraction of biochar, the presence and amount of the other elements is a function of the degree of thermal processing and type of feedstock utilized (Chen et al. 2019). As presented previously for feedstock analysis, proximate and ultimate analyses are usually carried out to investigate the chemical and elemental composition of biochar. Concerning mineral composition, this is carried out by melting and digestion of biochar ashes, followed by measurement using inductively coupled plasma optical emission spectrometry (ICP-OES) or inductively coupled plasma mass spectrometry (ICP-MS) according to DIN 51729, DIN EN ISO 11885, DIN EN ISO 17294-2 (EBC 2012).

Surface chemistry is a prominent determinant of biochar functional properties and defines how it interacts with the surrounding environment. The presence of various surface functional groups determines the biochar's sorption performance, hydrophobicity or hydrophilicity, $\mathrm{pH}$ buffering potential and polarity, which defines its ion exchange capacity (Chen et al. 2019). The presence of oxygen-containing functional groups and the concentration and distribution of such groups play a major role in defining the biochar's functional capabilities and specifically its sorption performance. Acidic functional groups, such as carboxyl, phenol and anhydride, have the highest impact on characteristics such as acidity, negative surface charge, hydrophilicity and general catalytic, electrical and chemical reactivity of the biochar (Usevičiūtè and Baltrènaitè-Gedienè 2020). At high processing temperatures, the presence of oxygen-containing and acidic functional groups decreases, while the presence of alkaline functional groups is enhanced (Chen et al. 2019).

Most functional groups containing hydrogen, oxygen and nitrogen are volatilized during the pyrolytic process, creating aromatic carbon surfaces. At this stage, the carbon is non-polar and hydrophobic; however, as biochar surface is exposed to the surrounding environment, carbon undergoes oxidation. This allows new oxygen-containing aromatic functional groups, e.g. carboxylic acid, carbonyls and hydroxyls, to be created, once again triggering polarity (CE 2012). Type of feedstock and pyrolytic processing conditions play a major role in defining the presence and density of the various functional groups found in the resulting biochar. Surface functional groups for biochar can be investigated using Fourier transform infrared spectroscopy (FTIR), as presented previously for feedstock analysis.

The $\mathrm{pH}$ values of biochar typically tend to be neutral to alkaline, and alkalinity is usually enhanced under elevated thermal processing temperatures, presenting an opportunity to be utilized as a liming agent (Chen et al. 2019). Higher alkalinity is attributed to the decomposition of acidic functional groups under high temperatures (Kazemi Shariat Panahi et al. 2020). Biochar $\mathrm{pH}$ is mainly dependant on the original feedstock as well as processing temperature. Inorganic mineral content in the feedstock plays a role in defining the degree of acidity in the resulting biochar. Feedstocks with low ash content, such as wood, tend to deliver biochar characterized by a lower $\mathrm{pH}$ value compared to feedstocks with higher ash fractions, when processed under the same conditions (Singh et al. 2017). The measurement of biochar $\mathrm{pH}$ can be carried out using a $\mathrm{pH}$ meter, according to ASTM-D4972 (Yargicoglu et al. 2015).

Electrical conductivity is a measurement of the amount of soluble salt in a biochar solution, and it is based on the principle that solutions with an elevated concentration of salts convey a better ability to conduct electrical current. This is an important characteristic depending on the final application of the biochar. For example, for agronomic applications, a high electrical conductivity value indicates the presence of salts, where a high biochar application rate may have negative effects on salt-sensitive plants (Singh et al. 2017). However, a high electrical conductivity value would be favourable for other applications such as supercapacitors for energy storage (Gabhi et al. 2020; Abdel Maksoud et al. 2020). Similar to $\mathrm{pH}$, the type of feedstock and the processing conditions are determinants for the electrical conductivity values measured in the resulting biochar. The presence of inorganic minerals and elevated pyrolytic temperatures tend to produce biochar with high electrical conductivity 
values (Singh et al. 2017). Electrical conductivity values can be measured using an electrical conductivity meter following the same preparation methods used for $\mathrm{pH}$ measurement (Yargicoglu et al. 2015).

As explained, the capacity of biochar for sorption and retention of ionic compounds such as nutrients and contaminants is defined by the biochar's surface chemistry. Surface charge in biochar is an important electrochemical property and is usually defined by certain functional groups. Sorption and retention of nutrients and heavy metals are primarily carried out via cation and anion exchange reactions which require the biochar surface to carry negatively charged and positively charged functional groups, respectively (Banik et al. 2018). Cation exchange capacity is an important chemical property reported in the literature especially for agronomic applications since it defines a biochar's ability to chemically attract and retain important nutrients such as $\mathrm{K}^{+}$, $\mathrm{Ca}^{2+}, \mathrm{Mg}^{2+}, \mathrm{H}^{+}, \mathrm{Na}^{+}$and $\mathrm{NH}_{4}{ }^{+}$. Carboxylate and phenolate functional groups are reported to mainly be responsible for the negatively charged sites found on the surface of biochar (Mia et al. 2017). Banik et al. in their investigation reported that biochar processed at temperatures below $500{ }^{\circ} \mathrm{C}$ shows high cation exchange capacity values and is optimized for cationic compound sorption. On the other hand, processing temperatures above $700{ }^{\circ} \mathrm{C}$ promote low cation exchange capacity values and high anion exchange capacity values, with the evident dominance of a positively charged surface due to the presence of non-hydrolyzable bridging oxonium functional groups (Banik et al. 2018).

The determination of cation exchange capacity is very challenging, where values reported in the literature show high variability and low reproducibility. The main challenge is that cation exchange capacity results mainly depend on the analysis method (Munera-Echeverri et al. 2018). Another measure for surface charge reported in the literature is zeta potential. Zeta potential is an interesting analytical technique that is used for the evaluation of surface charge of biochar. A zeta potential analyser is usually employed for carrying out measurements.

Polycyclic aromatic hydrocarbons are a group of organic contaminants formed on the surface of biochar during the production process. Polycyclic aromatic hydrocarbons are mainly caused by the incomplete combustion of biomass and their presence in the environment poses an elevated risk to human health (Henner et al. 1997; Wang et al. 2017; Lawal 2017; De la Rosa et al. 2019). Polycyclic aromatic hydrocarbons are aromatic structures that consist of linkages of two or more carbon rings, only containing carbon and hydrogen. The literature reports 16 major polycyclic aromatic hydrocarbons regulated and associated with a high toxicity level, as shown in Fig. 2 (Buss et al. 2016; de Resende et al. 2018). Besides the total concentration, the concentration of bioavailable polycyclic aromatic hydrocarbons is more critical in terms of risk. The type of feedstock and processing conditions are determinants of formation. In general, low molecular weight polycyclic aromatic hydrocarbons are formed under processing temperatures below $500{ }^{\circ} \mathrm{C}$, while high molecular weight polycyclic aromatic hydrocarbons are formed at higher temperatures, above $500{ }^{\circ} \mathrm{C}$ (Wang et al. 2017).

Furthermore, the literature reports that the pyrolytic process has an impact on the formation of polycyclic aromatic hydrocarbons, where biochar produced via slow pyrolysis carries lower concentrations compared to those produced via fast pyrolysis or gasification (Wang et al. 2017; Buss et al. 2016; Hale et al. 2012). Hale et al. investigated the total and bioavailable concentrations of 50 biochar samples prepared via slow pyrolysis. The total concentrations ranged from 0.07 to $3.27 \mu \mathrm{g} \mathrm{g}^{-1}$, while the concentrations of the bioavailable fraction ranged from 0.17 to $10 \mathrm{ng} \mathrm{L}^{-1}$, meeting environmental regulations; certification and regulation requirements will be further discussed in "Carbon removal marketplace participation requirements" section. However, biochar produced via gasification showed a total concentration of $45 \mu \mathrm{g} \mathrm{g}^{-1}$ and $162 \pm 71 \mathrm{ng} \mathrm{L}^{-1}$ for total and bioavailable concentrations, respectively, which exceeded regulation thresholds. In general, the concentrations of total and bioavailable polycyclic aromatic hydrocarbons varied according to the type of feedstock, pyrolysis temperature, residence time and thermochemical conversion technology utilized. Furthermore, the formation of other contaminants, such as polychlorinated dibenzodioxins and polychlorinated dibenzofurans, is possible during biochar production.

Total concentrations of polycyclic aromatic hydrocarbons can be determined via Soxhlet extraction using toluene followed by gas chromatography-mass spectrometry (GC-MS), according to DIN EN 15527:2008-9 (EBC 2012). Furthermore, polyoxymethylene passive samplers followed by GC-MS analysis can be employed for the quantification of bioavailable polycyclic aromatic hydrocarbons concentrations. Detailed protocols for total and bioavailable concentration determination are presented by Hale et al. (2012). The presence of heavy metals within the biochar matrix also poses other ecological risks and must be considered. Furthermore, environmental regulations dictate concentration thresholds for trace metals; this will be further explored in "Carbon removal marketplace participation requirements" section. Heavy metals include lead, cadmium, copper, nickel, mercury, zinc, chromium, boron, manganese and arsenic. Heavy metal concentrations can be determined via micro-wave assisted digestion followed by ICP-OES or ICP-MS analysis according to DIN 22022-2, DIN22022-7, DIN EN ISO 17294-2, DIN EN 1482 (EBC 2012). 


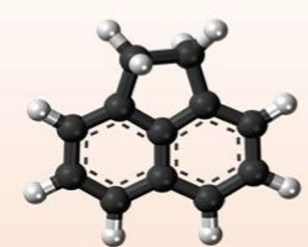

Acenaphthene

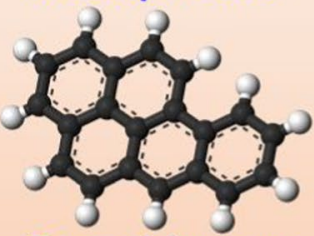

Benzo(a)pyrene

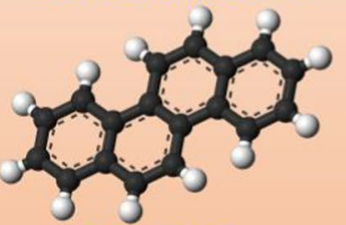

Chrysene

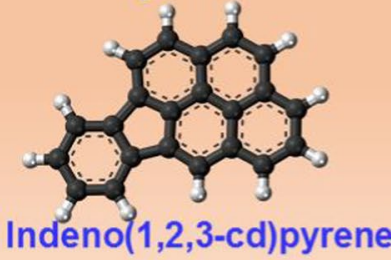

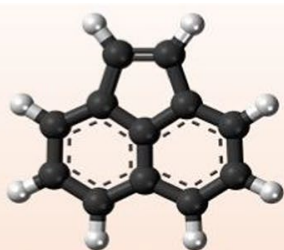

Acenaphthylene

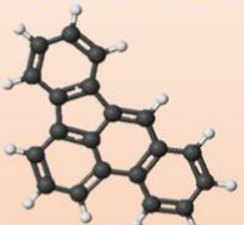

Benzo(b)fluoranthene

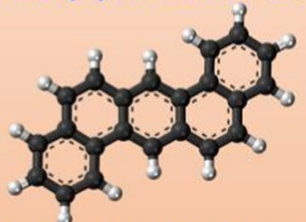

Dibenz(a,h)anthracene

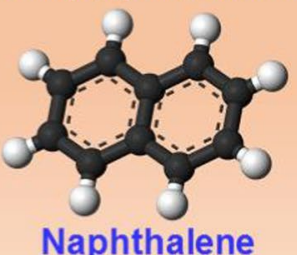

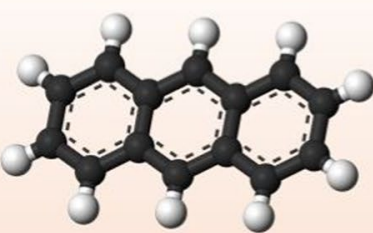

Anthracene

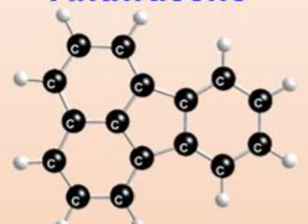

Benzo(k)fluoranthene

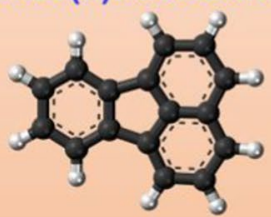

Fluoranthene

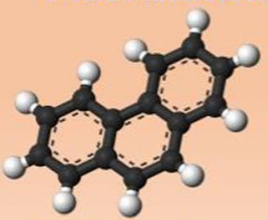

Phenanthrene

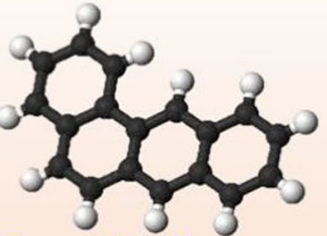

Benz(a)anthracene

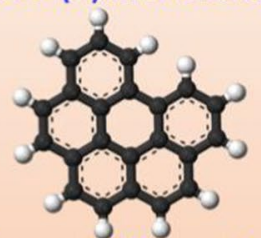

Benzo(ghi)perylene

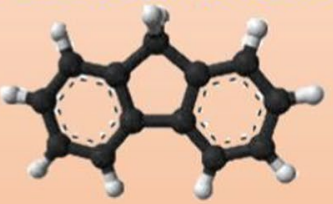

Fluorene

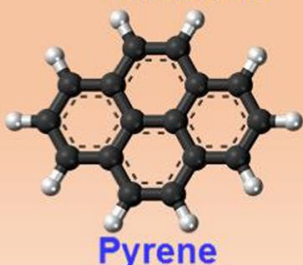

Fig. 2 Major sixteen polycyclic aromatic hydrocarbons that are regulated due to toxicity potential. Polycyclic aromatic hydrocarbons consist of multiple aromatic rings. They are mainly caused by the incomplete combustion of biomass and are usually formed on the surface of biochar

\section{Physical and structural properties}

Biochar physical and structural properties are also directly related to the physical properties associated with the original feedstock and the degree of pyrolytic processing conditions carried out. The most prominent physical properties related to biochar that are reported in the literature include density, particle size, mechanical strength and most importantly surface structure, which constitutes surface area and porosity. These properties have an influencing role in defining the functional capabilities of biochar for various applications.

Biochar particle size is a function of the original feedstock particle size and the degree of thermal processing. In general, the size obtained in the resulting biochar after pyrolytic conversion of raw biomass is usually smaller than the original particle size. This is a result of the shrinkage and particle attrition that takes place during pyrolysis. However, in some cases, agglomeration might take place, resulting in larger particle sizes. Furthermore, there is evidence that at higher processing temperatures, particle size tends to be smaller. This can be explained by the fact that tensile strength decreases at higher temperatures, which lowers attrition resistance (Kazemi Shariat Panahi et al. 2020;
Downie et al. 2009). Particle size is an important physical characteristic as it defines the suitability and performance of biochar in various applications. Particle size distribution can be determined using sieving or image particle analysis as previously discussed under feedstock analysis.

Biochar density types include solid density as well as bulk density. The solid density represents the density on a molecular level which is related to the extent to which the carbon structure has been packed as a result of the loss of volatile and condensable compounds and the consequent formation of graphite-like crystallites. Solid density, therefore, increases under higher processing temperatures. Typical values of solid densities reported in the literature range between 1.5 and $1.7 \mathrm{~g} \mathrm{~cm}^{-3}$. On the other hand, bulk density involves the density of multiple particles, including the voids present in between the particles. Biochar derived from wood typically has bulk densities ranging from 0.3 to $0.43 \mathrm{~g} \mathrm{~cm}^{-3}$ (Mulabagal et al. 2017; Kazemi Shariat Panahi et al. 2020; Downie et al. 2009). Mechanical strength of biochar is directly related to its solid density and its degree of aromaticity and molecular order. Mechanical strength is an important attribute as it directly influences the biochar's ability to withstand physical stresses within various 
applications. In general, feedstocks with high lignin content and low ash content have shown good mechanical properties when converted to biochar (Kazemi Shariat Panahi et al. 2020; Downie et al. 2009).

Surface structure and in particular pore-size distribution is perhaps one of the very important aspects in relation to biochar's adsorptive performance. Pores are generally classified into three distinct groups, namely micropores, mesopores and macropores with internal diameters of lower than $2 \mathrm{~nm}$, 2-50 nm, and higher than $50 \mathrm{~nm}$, respectively. Micropores highly contribute to biochar surface area and have the highest impact on adsorptive performance, as they mainly target minute molecules, e.g. gases and solvents. Mesopores, on the other hand, are critical in many liquid-solid adsorption processes. Macropores are important as they act as channels for adsorbates to reach micro- and mesopores. Furthermore, in agronomic applications, macropores play a vital role in facilitating plant root movement, soil aeration, hydrology and facilitating a habitat for microbial communities (Kazemi Shariat Panahi et al. 2020; Downie et al. 2009).

The literature reports the specific surface area for biochar to be in the range of $1.5-500 \mathrm{~m}^{2} \mathrm{~g}^{-1}$, with higher temperatures generally leading to enhanced microporosity as well as larger surface areas. During pyrolytic reactions at low temperatures, volatiles and tars usually fill up and block the internal pores of the biochar leading to small surface areas. However, as temperature is elevated, these substances are volatilized, leading to enhanced microporous structures and larger surface areas. At certain critical temperatures, micropore destruction starts to take place and surface area starts to decline (Chen et al. 2019). This turning point is usually feedstock and condition specific, and generally occurs at temperatures higher than $850{ }^{\circ} \mathrm{C}$ (Kazemi Shariat Panahi et al. 2020). Moreover, biochar surface area can be further enlarged to enhance its adsorptive capabilities; however, this requires consequent physical or chemical modifications which will be further discussed in "Biochar engineering" section. The specific surface area and pore size and volume are usually determined and evaluated using the Brunauer-Emmett-Teller nitrogen physisorption method, following DIN ISO 9277 (EBC 2012). Furthermore, scanning electron microscopy is usually carried out to qualitatively investigate the surface morphology of the biochar.

\section{Hydrological properties}

Biochar's hydrological properties are important in relation to agronomic applications, where the addition of biochar to soils can potentially improve water retention. Two distinct water retention-related properties are discussed in the literature, namely water holding capacity and wettability. Water holding capacity is a measure of the amount of water retained within biochar and soil micro-and mesopore structures, which is available for plant uptake. The water holding capacity is mainly influenced by porosity, surface area and the presence of oxygen-containing surface functional groups and the resulting surface charge (Ulusal et al. 2020; Batista et al. 2018). Wettability, on the other hand, is a function of the degree of surface hydrophobicity. At low pyrolysis temperatures, biochar tends to be hydrophobic, which is explained by the presence of aliphatic compounds on the biochar surface. At elevated processing temperatures, such compounds are volatilized, promoting a more hydrophilic state. It is important to note that to produce biochar with enhanced water retention properties, sufficient porosity and minimum hydrophobicity are desired, which can be controlled by the selection of appropriate feedstock and choice of pyrolysis temperature (Usevičiūtè and BaltrenaitèGedienė 2020; Kinney et al. 2012; Kameyama et al. 2019; Gray et al. 2014).

Water retention potential of biochar needs to be assessed when biochar is applied and mixed with soil since biochar-soil dynamics need to be taken into account for a realistic evaluation. Kinney et al. in their investigation identified that the trend of water holding capacity for pure biochar was not consistent with that of biochar-soil mixtures in sandy soils, where pure biochar showed a range of capacities from 0.77 to $11.1 \mathrm{~g} \mathrm{~g}^{-1}$. A tenfold variation in capacity was observed in samples prepared from three types of feedstocks under various processing temperatures. However, when mixed with soil, only a modest $24-36 \%$ increase in capacity was achieved compared to a control plot without biochar (Kinney et al. 2012).

Water holding capacity can be determined in accordance with DIN EN ISO 14238. The test includes soaking a $2 \mathrm{~mm}$ fraction of the material in water for $24 \mathrm{~h}$. Consequently, the material is placed on a dry sand bed to remove excess water for $2 \mathrm{~h}$. Furthermore, the saturated biochar is weighed and then dried at $40{ }^{\circ} \mathrm{C}$. The material is then weighed again, post-drying to determine the amount of retained water (EBC 2012). In terms of hydrophobicity, various techniques have been discussed in the literature, namely water drop penetration test, the molarity of ethanol drop test and contact angle measurement (Kinney et al. 2012; Usevičiūté and Baltrénaité 2020).

\section{Carbon stability}

\section{Properties related to carbon stability}

Perhaps the most important property defining biochar's stability is its aromatic carbon structure, which includes both amorphous and crystalline phases represented by randomly organized aromatic rings and condensed polyaromatic sheets, respectively. Aromaticity and the degree of aromatic condensation define the extent of carbon stability 
within biochar. A high level of aromaticity and degree of aromatic condensation indicates that the biochar is likely to resist thermochemical and biological decomposition, showing stability. Another important aspect is the reactivity of the carbon structure, where the presence of various oxygenand nitrogen-containing functional groups and non-aromatic carbon structures, which constitute the labile fraction within biochar, negatively influence stability by enhancing vulnerability to degradation (Leng et al. 2019a; Leng and Huang 2018).

Furthermore, the elemental composition of biochar is indicative of carbon structure and degree of carbon stability. Since nitrogen makes up a very small fraction of biochar, carbon, hydrogen and oxygen constitute the major compositional elements. The availability of oxygen is closely related to surface functional groups that influence reactivity and enhance degradation potential. Moreover, the relationship between the amount of hydrogen and carbon available within biochar indicates the degree to which fused aromatic ring structures have been created, which is a proxy for the degree of aromaticity (Leng et al. 2019a; Leng and Huang 2018). Furthermore, other properties have been reported in the literature affecting the stability of carbon in soils, such as inorganic content, pore structure, surface area, $\mathrm{pH}$ and particle size (Leng and Huang 2018; Lehmann et al. 2011). In relation to particle size, it has been reported that fine particles are more susceptible to microbial attack (Bruun et al. 2012).

\section{Carbon stability assessment techniques}

Three main routes are reported in the literature in relation to the assessment of carbon stability. The first route constitutes methods that carry out carbon structure analysis. The second assessment route considers the oxidation resistance of biochar by analysing the labile and stable carbon fractions. Finally, the third route involves carrying out incubation and modelling methods to analyse stability while considering biotic and abiotic degradation effects.

Several techniques can be utilized to analyse carbon structure, and the simplest method is the ultimate analysis. The molar $\mathrm{H} / \mathrm{C}_{\text {org }}$ and $\mathrm{O} / \mathrm{C}_{\text {org }}$ ratios are the most prominent proxies for carbon stability reported within the literature and utilized by biochar certification bodies in regulating biochar stability. Lower values for both ratios indicate stable carbon that can resist thermal and biological degradation. Spokas reviewed the stability of biochar in soils and suggested that biochar with a molar $\mathrm{O} / \mathrm{C}_{\text {org }}$ ratio below 0.2 appeared to exhibit a half-life of at least 1000 years, while biochar with a value between 0.2 and 0.6 has a half-life between 100 and 1000 years. Values above 0.6 indicate a half-life below 100 years (Spokas 2010). The international biochar initiative as well the European biochar certificate both place a regulatory $\mathrm{H} / \mathrm{C}_{\text {org }}$ limit at a value of 0.7 , while the European biochar certificate places an $\mathrm{O} / \mathrm{C}_{\text {org }}$ limit at a value of 0.4 (EBC 2012; IBI 2015). Furthermore, instrumental methods can be utilized for carbon structure analysis such as nuclear magnetic resonance, Raman spectroscopy, mid-infrared spectroscopy, X-ray diffraction, high-resolution transmission electron microscopy (HRTEM), near-edge X-ray absorption fine structure spectroscopy (NEXAFS) and X-ray photoelectron spectroscopy (Wiedemeier et al. 2015; Calvelo Pereira et al. 2011). Furthermore, molecular markers such as polycyclic aromatic hydrocarbons, benzene polycarboxylic acid, n-alkanes, lipid analysis and markers from pyrolysis-gas chromatography-mass spectrometry (Py-GC-MS) as well as skeletal density analysis via helium pycnometry are all indicators of carbon structure (Wiedemeier et al. 2015; Calvelo Pereira et al. 2011).

The second assessment route considers external elements that may influence stability, where oxidation resistance of biochar is quantified by analysing the labile and stable carbon fractions. A number of methods are reported in the literature, namely proximate analysis, and thermal degradation techniques such as temperature-programmed oxidation and the recalcitrant index $\left(\mathrm{R}_{50}\right)$ introduced by Harvey et al. (Harvey et al. 2012). Furthermore, chemical oxidation using hydrogen peroxide $\left(\mathrm{H}_{2} \mathrm{O}_{2}\right)$ with heat treatment (Cross and Sohi 2013), as well as potassium dichromate $\left(\mathrm{K}_{2} \mathrm{Cr}_{2} \mathrm{O}_{7}\right)$ (Knicker et al. 2007; Liu et al. 2020), and finally permanganate $\left(\mathrm{KMnO}_{4}\right)($ Calvelo Pereira et al. 2011) have been reported. The oxidation techniques mainly focus on removing the labile carbon fraction to quantify stability.

The third route involves incubation and modelling experiments, and this is perhaps the most important assessment technique to directly study mineralization behaviour and carbon stability, as it adequately considers biotic and abiotic degradation. The results obtained via most of the methodologies discussed in the first and second routes are usually correlated with incubation and modelling techniques and are presented as proxies. Stability assessment via this route involves applying biochar into the soil and measuring the assays of $\mathrm{CO}_{2}$ that evolve during incubation, or determining the amount of carbon that remains in the soil over the experimental period. Since biochar may persist in the soil for hundreds or thousands of years, it is not feasible to carry out such assessments. This is, however, overcome by carrying out modelling based on the mineralization data obtained via incubation.

Leng et al. discuss various modelling techniques, namely one-pool, two-pool, three-pool and logarithmic models. These models are employed to estimate the mean residence time or half-life of biochar. Although this is considered as the most realistic approach for stability assessment, it carries various drawbacks. Leng et al. discuss that the duration of incubation, the method utilized for determining mineralization, the selection of models, fitting procedures and the 
incubation environment are all aspects that introduce significant variation to the stability results obtained. To obtain high accuracy results, Leng et al. recommend that the incubation period is lengthened, a double exponential model should be employed, carbon isotopic technology to be utilized for measuring $\mathrm{CO}_{2}$ evolution, to carry out model fitting using the percentage of total organic carbon mineralized, and finally it is preferred that the biochar is applied in the field (Leng et al. 2019b).

For industrial applications, stability assessment should be accurate, fast and cost-effective. Carbon stability assessment via carbon structure analysis is interesting; however, various drawbacks are noted, mainly the high cost and inaccessibility or lack of operational know-how to most of the analytical instruments required. However, the $\mathrm{H} / \mathrm{C}_{\text {org }}$ and $\mathrm{O} / \mathrm{C}_{\text {org }}$ indices seem to be the most feasible proxies. On the other hand, incubation and modelling require long experimental periods and special setups that are costly and are not feasible to carry out. This approach, if carried out properly, should be used for correlation with other proxies. While most of the oxidation resistance techniques are easy to carry out, and costs can be maintained, further validation and correlation with incubation experiments are required. For example, the Edinburgh stability tool introduced by Cross and Sohi involves chemical oxidation using $0.01 \mathrm{M} \mathrm{H}_{2} \mathrm{O}_{2}$ at a temperature of $80{ }^{\circ} \mathrm{C}$ for two days; the process is regarded as a proxy for biochar weathering over 100 years. However, the drawback to this approach is the lack of validation by corresponding incubation tests (Liu et al. 2020).

Recently, Liu et al. proposed a fast and cost-effective chemical oxidation method for predicting long-term biochar mineralization in soils. The protocol utilizes $0.1 \mathrm{M} \mathrm{K}_{2} \mathrm{Cr}_{2} \mathrm{O}_{7}$ and $0.2 \mathrm{M} \mathrm{H}^{+}$at a temperature of $100{ }^{\circ} \mathrm{C}$ for $2 \mathrm{~h}$. The experimental work showed that the labile carbon fraction oxidized was highly correlated, with an $\mathrm{R}^{2}$ value higher than 0.99 , with one that would be mineralized in the soil at a scale of 100 years (Liu et al. 2020). While this method probably requires further validation, for example, using more types of biochar and applied to fields as opposed to the laboratory, this is perhaps the most promising stability assessment technique in terms of cost, accuracy and preparation time required, reported in the literature thus far.

\section{Effect of feedstock and process parameters on biochar yield and properties}

As previously discussed, the type of feedstock utilized and the specified processing conditions define the properties and quality of the resulting biochar as well as influence production yield. This section will further explore the effect of various parameters: type of feedstock, processing temperature, residence time, particle size, heating rate, carrier gas, pressure, various modification techniques, and other parameters on biochar yield and properties and the general efficiency of the pyrolytic conversion process. Figure 3 illustrates the major parameters that influence biochar yield and properties.

\section{Type of feedstock}

Feedstock selection plays a critical role in defining the properties, composition and yield of the resulting biochar. The composition, structure and characteristics of the original feedstock significantly influence the nature of the pyrolytic product. In general, feedstocks can be categorized into lignocellulosic and non-lignocellulosic materials. Each of these materials carries a relative mass ratio of organic and inorganic fractions that vary with the biomass's type and growing conditions (Kan et al. 2016; Qambrani et al. 2017). In general, biomass with a high inorganic fraction, such as manures and bio-solids, is characterized with low surface area and porosity, high cation exchange capacity and $\mathrm{pH}$, high nutrient availability (Ippolito et al. 2020), and high solid fraction yield which is mainly due to the catalytic effect of the inorganic fraction in the parent material. However, such feedstocks produce biochar with lower carbon content and stability (Kumar et al. 2020). The stability is highly influenced by the reactive nature of the inorganic fraction (Leng and Huang 2018).

Furthermore, lignocellulosic materials, either woody or herbaceous, are mainly composed of varying degrees of cellulose, hemicellulose, lignin and inorganic matter. As previously discussed, pyrolysis of each of the main constituents follows unique reaction pathways that highly influence the yield and characteristics of the resulting biochar. The literature reports that lignin is the major contributor to the solid fraction yield and that biomass with high lignin content usually exhibits high biochar yield, high carbon content, high specific area and a more aromatic carbon structure ( $\mathrm{Li}$ et al. 2020; Jiang et al. 2020), favouring long-term stability. Wood-based biochar typically exhibits high surface area and porosity, low $\mathrm{pH}$ and cation exchange capacity, high carbon content and lower nutrient concentrations compared to herbaceous and non-lignocellulosic materials (Ippolito et al. 2020). The high lignin content and low inorganic mineral fraction in woody material are the main contributors to carbon stability in the resulting biochar (Leng and Huang 2018). For carbon sequestration applications, wood-based biochar exhibits the highest carbon removal and stability potential (Ippolito et al. 2020). In relation to herbaceous materials, the varying structure, in terms of cellulose, hemicellulose, lignin and the amount of inorganic minerals present, defines the resulting physicochemical and 


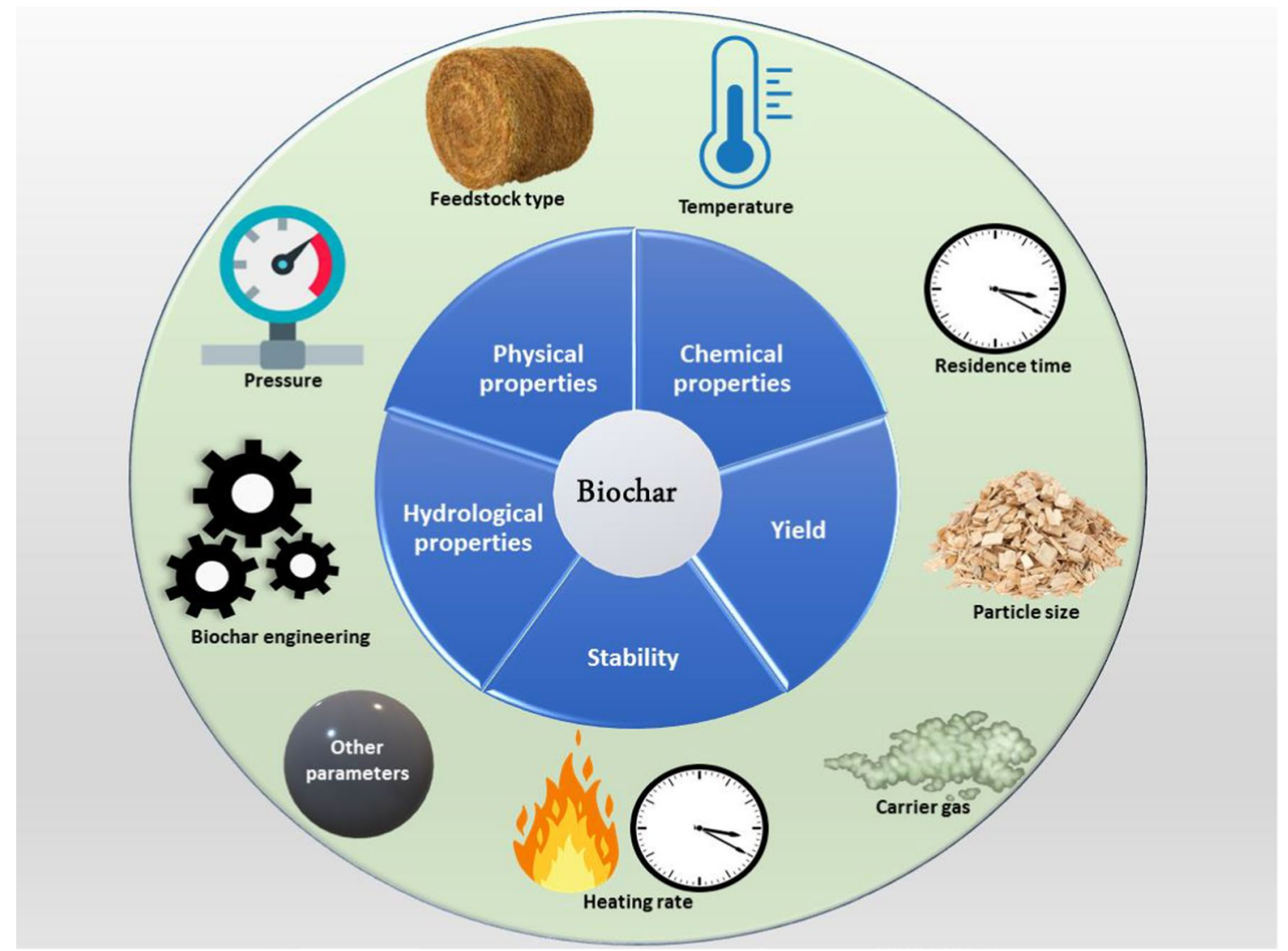

Fig. 3 Major parameters influencing biochar yield, stability and general physical, chemical and hydrological properties. This includes feedstock type, processing temperature, residence time, particle size, carrier gas, heating rate, pressure, engineering techniques as well as other parameters that may have an overall impact on the efficiency of the conversion process stability properties of the biochar. However, herbaceous materials typically result in biochar with properties that fall in between wood-based and non-lignocellulosic-based biochar (Ippolito et al. 2020).

The impact of biomass type on the formation of polycyclic aromatic hydrocarbons during pyrolysis has been investigated and reported in the literature. General findings suggest that herbaceous-based biochars tend to contain higher concentrations of polycyclic aromatic hydrocarbons compared to wood-based biochar. Buss et al. reported a 5.8 times higher concentration of straw-derived biochar compared to wood-based (Buss et al. 2016). Keiluweit et al. shared a similar finding regarding grassbased biochar exhibiting a concentration that is four times higher than wood-derived biochar (Keiluweit et al. 2012). Furthermore, other studies report similar findings (Kloss et al. 2012; Fabbri et al. 2013). It has been observed that feedstocks rich in lignin tend to produce biochars with low polycyclic aromatic hydrocarbons concentrations
(Buss et al. 2016); however, the literature presents mixed results on this matter (Zhou et al. 2014).

\section{Temperature}

Besides the choice of feedstock, process temperature is by far the most prominent production parameter that significantly influences yield, stability and physicochemical properties of the resulting biochar. The impact of temperature is well documented in the literature. Biochar yield is negatively correlated with temperature; that is at higher processing temperatures a decrease in biochar yield is realized (Kan et al. 2016; Demirbas 2004; Williams and Nugranad 2000; Shabangu et al. 2014). Furthermore, in terms of physical and structural properties, temperature is positively correlated with surface area, porosity, bulk density and mechanical strength. At higher temperatures, the release of volatiles opens up the pores and enhances porosity in general leading to enlarged surface areas and enhanced adsorptive performance (Chun et al. 2004; Chen 
et al. 2008). However, this is achieved up to a critical point, whereby high temperatures contribute to the deformation and collapse of the pore structure, which reduces the specific surface area (Li et al. 2020). As previously discussed, temperature significantly influences the chemical properties of the biochar. At higher processing temperatures, the reduction of various oxygen-containing surface functional groups, such as hydroxyl, carboxyl, carbonyl, lactone and ether, is observed (Várhegyi et al. 1998), which ultimately leads to a lower capacity for cation exchange, a higher $\mathrm{pH}$ and a decrease in surface charge (Tomczyk et al. 2020). Electrical conductivity has also been observed to be positively correlated with temperature (Singh et al. 2017).

In terms of hydrological properties, water holding capacity and hydrophilicity tend to increase along with temperature. Water holding capacity is a function of the presence of a microporous structure and large surface area, and these two properties are generally enhanced under elevated temperatures. Furthermore, wettability is highly influenced by the volatilization of aliphatic compounds from the biochar surface and this usually takes place at higher temperatures. In general, the literature reports a positive correlation between water holding capacity and wettability with processing temperature (Usevičiūtè and Baltrènaitè-Gedienè 2020; Kinney et al. 2012; Kameyama et al. 2019; Gray et al. 2014).

In terms of elemental composition, it has been observed that elevated temperatures are conducive of biochar that is characterized with high carbon and low hydrogen and oxygen contents. Moreover, ash content is also positively correlated with temperature. In relation to carbon stability, temperature is the most significant determinant of aromaticity and aromatic condensation. The literature reports a positive relationship between temperature and the presence and amount of stable fused aromatic carbon structures and the degree of aromatic condensation. A trade-off between biochar yield and carbon stability is observed and must be taken into account when optimizing production parameters (Leng and Huang 2018). Stable carbon yield by mass should, therefore, be the optimized factor (biochar yield wt.\% * stable carbon fraction wt.\%).

The impact of temperature on polycyclic aromatic hydrocarbons concentrations in biochar has been inconclusive so far within the literature. While various studies reported decreasing concentrations with increasing temperatures (Brown et al. 2006; Freddo et al. 2012), other studies found the opposite (Rogovska et al. 2012; Zielińska and Oleszczuk 2015), as well as no dependence (Kloss et al. 2012). Buss et al. carried out an investigation using 46 biochars prepared under controlled pyrolytic conditions and concluded that temperature alone is not the main influencing factor behind polycyclic aromatic hydrocarbons concentrations and that temperature along with other process conditions should be taken into account to explain the relationship between processing conditions and such concentrations (Buss et al. 2016).

\section{Residence time}

Reaction residence time is an important parameter influencing biochar yield, stability, physical structure, and overall production throughput and efficiency. However, it is important to note that the degree of influence is based on other process parameters, mainly temperature. At low processing temperatures, residence time is critical to the effectiveness of the pyrolytic conversion and the stability of the resulting biochar. However, at temperatures above $550{ }^{\circ} \mathrm{C}$ the impact of residence time becomes insignificant (Cross and Sohi 2013). It has been reported in the literature that longer residence periods provide sufficient time for the creation of microporous structures and enhanced surface area. At low pyrolytic temperatures, a long residence time usually leads to enhanced biochar yields. This is mainly caused by the repolymerization and condensation reactions taking place between the solids and vapours produced (Yaashikaa et al. 2019).

The impact of residence time on polycyclic aromatic hydrocarbons concentrations has been discussed in the literature, where a major effect is observed within fast pyrolysis. However, under slow pyrolysis, residence time had an insignificant effect. This, however, was based on experiments carried out in a batch reactor. The impact of residence time on such concentrations using a continuous system needs to be further investigated (Buss et al. 2016). In general, residence time plays a critical role in defining production throughput and production profitability; therefore, a trade-off exists between yield, required biochar characteristics and production throughput. Therefore, process optimization is required to achieve the optimum residence time that can ensure high yields, high productivity, as well as achieve the required product properties.

\section{Particle size}

Particle size is also another important factor influencing biochar yield as well as production throughput. Particle size mainly influences mass and heat transfer rates which define the degree and rate of volatilization. A larger particle size usually favours char formation. The impact of particle size on biochar yield has been investigated widely in the literature. Most studies report a positive correlation between particle size and biochar yield, which applies to both slow and fast pyrolysis (Demirbas 2004; Mani et al. 2010; Choi et al. 2012a).

Demirbas explored the impact of particle size on pyrolytic product yields using corn cob and olive husk at a pyrolysis temperature of $677^{\circ} \mathrm{C}$. The results indicated a significant 
increase in biochar yield from $5.7-16.6 \%$ to $19.4-35.6 \%$ for corn cob and olive husk, associated with the increase in particle size from 0.5 to $2.2 \mathrm{~mm}$ (Demirbas 2004). Choi et al. investigated the fast pyrolysis of wheat straw at $480{ }^{\circ} \mathrm{C}$ and explored the impact of various process conditions on product yield distribution. A modest increase in biochar yield from 17.7 to $19.6 \%$ was observed, when particle size increased from 0.5 to $2.5 \mathrm{~mm}$ (Choi et al. 2012a). Zhang et al. also reported an increase in biochar yield from 22.2 to $28 \%$ while increasing particle size from a $0.5-1$ to $3-4 \mathrm{~mm}$, in line with the literature (Zhang et al. 2009). However, Encinar et al. have reported insignificant changes in biochar yield associated with increases in particle size (Encinar et al. 2000).

Furthermore, particle size significantly influences bulk density, which consequently impacts production throughput. Since most pyrolysis reactors are limited by volumetric capacity, bulk density is critical in defining the amount of feedstock that can be processed within a specific time frame. Optimization needs to be carried out to determine the particle size that would provide the highest yield at the highest production throughput possible.

\section{Heating rate}

Heating rate is a fundamental factor that defines the type of pyrolytic conversion process undertaken, e.g. slow, fast or flash pyrolysis, and the product yield distribution. Fast heating rates tend to enhance the fragmentation and depolymerization of biomass into primary volatiles, favouring the production of liquid and gaseous fractions and less char (Kan et al. 2016). This has been reported extensively within the literature, where oil yield is increased in line with faster heating rates; however, it has also been reported that after reaching specific heating rates further increases have an insignificant effect on oil yield (Onay 2007; Ozbay et al. 2006; Salehi et al. 2009). However, at lower heating rates, the degradation of the biomass is minimized during the secondary decomposition phase, which favours biochar production. Many studies reported the impact of low heating rates on enhanced biochar yield; Zeng et al. investigated the pyrolytic behaviour of beech wood under a temperature range of $600-2000{ }^{\circ} \mathrm{C}$ and heating rate range of $5-450{ }^{\circ} \mathrm{C} \mathrm{s}^{-1}$. Under all temperatures, a lower heating rate resulted in a higher biochar yield (Zeng et al. 2015).

Furthermore, Aysu and Kucuk reported similar results while investigating the pyrolytic behaviour of giant fennel stalks under a temperature range of $350-600{ }^{\circ} \mathrm{C}$ at heating rates of 15,30 and $50{ }^{\circ} \mathrm{C} \mathrm{min}{ }^{-1}$. At all temperatures investigated, the highest char yields were achieved at the lowest heating of $15{ }^{\circ} \mathrm{C} \mathrm{min}{ }^{-1}$ (Aysu and Küçük 2014). Other studies reported the same impact of heating rate on biochar yields (Angın 2013; Şensöz and Angın 2008). The impact of heating rate on carbon stability has also been reported in the literature. The formation of aromatic structures in biochar has been observed to be better facilitated at low heating rates as compared to high heating rates (Leng and Huang 2018).

Furthermore, it has been argued that low heating rates tend to facilitate the retention of structural complexity while at higher heating rates local melting, swelling and phase transition of the cell structures lead to the loss of structural complexity (Leng and Huang 2018; Cetin et al. 2004). The literature also reports that a low heating rate does not necessarily enhance biochar stability. An investigation reported no impact of a heating rate range of $1-100{ }^{\circ} \mathrm{C} \min ^{-1}$ on the stable carbon fraction, although at the higher heating rates a small decline was observed in the concentration of stable carbon (Crombie et al. 2013). Furthermore, in another study, there was no statistical significance on the impact of a heating rate range of 5-20 ${ }^{\circ} \mathrm{C} \mathrm{min}^{-1}$ on the stable carbon fraction, $\mathrm{O} / \mathrm{C}_{\text {org }}$ molar ratio, fixed carbon and volatile matter (Cross and Sohi 2013). In conjunction with temperature, low heating rates allow more time for the effect of temperature on biochar stability (Leng and Huang 2018). In general, lower heating rates are favourable for biochar production in terms of yield, structure and stability.

\section{Carrier gas}

The carrier gas is another parameter reported in the literature that can have an impact on biochar yield. A carrier gas, such as nitrogen or argon, is usually used to provide an inert environment for the conversion process. The gas itself does not participate in any reactions and is only utilized to flush the produced vapours outside the reaction zone. Therefore, an increased carrier gas rate would shorten the vapour residence time not allowing further repolymerization to take place and hence a lower char yield is obtained. The effect of increasing gas flow rate on biochar yield has been reported in many studies; however, the effect is usually marginal (Zhang et al. 2009; Ertaş and Hakk1 Alma 2010; Demiral and Ayan 2011; Heidari et al. 2014). Furthermore, the impact of carrier gas flow rate on the formation of polycyclic aromatic hydrocarbons has been investigated within the literature (Leng and Huang 2018). It is reported that the concentration of polycyclic aromatic hydrocarbons was reduced with an increase in gas flow rate, and such effect took place irrespective of processing temperature and residence time (Buss et al. 2016). In terms of carrier gas impact on stability, more research is required. In industrial pyrolysis systems aimed for biochar production, a carrier gas is usually not used, as long as the reactor is adequately sealed and an oxygen-free environment is maintained, and polycyclic aromatic hydrocarbons thresholds are not exceeded. For fast pyrolysis, 
this might not be the case as short vapour residence times are usually required to maximize oil yield, and therefore, the utilization of carrier gas is important.

\section{Pressure}

Pyrolytic conversion is usually carried out under atmospheric or low-pressure conditions; however, the impact of pressure on biochar yield, properties and stability has been investigated within the literature. The positive effect of pressure on biochar yield was first recognized by Mok and Antal in 1983 and was further confirmed by many researchers (Blackadder and Rensfelt 1985; Richard and Antal 1993; Antal et al. 1996; Mok and Antal 1983). Antal et al. investigated the impact of pressure on the yield of air-dried macadamia nutshell char and showed that at the same processing temperature, an increase in char yield from 40.5 to $51 \%$ was associated with an increase in pressure from 0.4 to $3.3 \mathrm{MPa}$ (Antal et al. 1996). Further results were shared in another publication showing the negative correlation between pressure on fixed carbon. As pressure was increased from 0.4 to $3.3 \mathrm{MPa}$, fixed carbon was reduced from 78.6 to $69.9 \%$; however, in general, an increase in fixed carbon yield was noted since biochar yield increased (Antal and Grønli 2003). The increase in yield associated with increasing pressure is mainly attributed to the higher concentration of vapour phase within the reactor, enhancing the repolymerization of volatiles, leading to further biochar production (Antal et al. 1996). However, the impact of pressure on the formation of polycyclic aromatic hydrocarbons should be taken into account. Matamba et al. carried out an investigation using flash pyrolysis of palm kernel shell at a temperature range of $600-900{ }^{\circ} \mathrm{C}$. While increased pressure enhanced biochar yield, higher concentrations of polycyclic aromatic hydrocarbons were generated (Matamba et al. 2020).

In terms of carbon stability, elevated pressure has increased aromatic carbon content in several studies. Melligan et al. investigated the impact of pressure, ranging from atmospheric to 26 bar, on the pyrolytic conversion of miscanthus in a batch reactor at $550{ }^{\circ} \mathrm{C}$. An increase in aromatic carbon content was observed and an increase in heating value; however, the yield was not significantly affected, and surface area was negatively impacted at higher pressures. The low surface areas obtained at high pressures are mainly explained by the fact that at higher pressures, the pore structure collapses and tar is trapped on the biochar surface (Melligan et al. 2011). Manya et al. investigated the slow pyrolysis of olive mill waste at a temperature range of $400-600{ }^{\circ} \mathrm{C}$ and pressure range of $0.1-1.1 \mathrm{MPa}$. The experimental results indicated a positive relationship between pressure and stability, as shown via the statistically significant impact of pressure on aromatic carbon content, fixed carbon content and $\mathrm{O} / \mathrm{C}_{\text {org }}$ molar ratios. However, the impact of pressure on biochar yield contradicted the general consensus, where higher pressures decreased the yields. The authors attribute this phenomenon to the significance of vapour phase residence time, and the intrinsic nature of the feedstock used (Manyà et al. 2014).

\section{Biochar engineering}

Biochar properties and characteristics can be further enhanced by carrying out a number of modification techniques, either pre- or post-pyrolytic processing. In general, biochar modification is carried out to enhance the surface area, modify surface characteristics by creating new functional groups and prepare new biochar matrix composites to meet specific application requirements (Liu et al. 2020). The literature reports a number of modification methods, each with specific impacts on physicochemical properties and functionality.

Activation is a very popular technique developed to enhance the surface area, porosity and surface characteristics, which significantly impacts the adsorptive performance. Generally, two routes are discussed within the literature, physical and chemical (Balajii and Niju 2019; Kosheleva et al. 2019; Heidarinejad et al. 2020). Physical activation is carried out at high temperatures in the presence of steam or gas such as $\mathrm{CO}_{2}$. The main issue with physical activation is the high energy requirement associated with the process. Chemical activation is carried out using acid or alkali reagents and is usually carried out at much lower pyrolytic temperatures $\left(450-550^{\circ} \mathrm{C}\right)$ which provides good yields and utilizes lower energy; however, the issue related to chemical activation relates to waste treatment requirements since chemicals are involved in the process.

Other modification methods reported in the literature include ultrasonication, electrochemical treatment, mineral oxide impregnation or coating, ball milling, plasma treatment and microwave treatment ( $\mathrm{Li}$ et al. 2020; Akhil et al. 2021; Kuppusamy 2019; Wang et al. 2018; Mudhoo et al. 2019; Gopinath and Kadirvelu 2018). Each of these treatment methods provides unique properties by introducing new physicochemical capabilities which ultimately enhance selective sorption performance. These modifications are very applicable to environmental remediation, wastewater treatment, catalysis and energy storage applications (Gopinath et al. 2021). Biological modification using microorganisms via anaerobic digestion or bacteria has also been reported to influence surface charge ( $\mathrm{Li}$ et al. 2020; Wang et al. 2018). Furthermore, nutrient loading through co-composting was reported to enhance slow nutrient release, which can be extremely beneficial in agronomic applications (Kuppusamy 2019). Functionalized biochar is a growing area of research; however, it is important to consider the impact of 
such engineering efforts on the stability of biochar if it is to serve the purpose of carbon sequestration in conjunction with the application for which the biochar has been functionalized for. As previously mentioned, functionalization increases reactivity which hinders the carbon sequestration potential that could be achieved.

\section{Other parameters}

Generally, pre-treatment of biomass feedstocks is required prior to pyrolytic conversion. The literature presents various categories for pre-treatment technologies, namely physical, thermal, chemical, biological and a combination of such technologies. Most of these technologies are used for enhancing the bio-oil fraction (Kan et al. 2016). However, pre-treatment is also necessary to enhance biochar properties and the overall efficiency of the conversion process. Depending on the feedstock used and the state at which the materials arrive to the conversion plant, the following pre-treatment processes may be required. Cleaning the feedstock is perhaps a critical step towards protecting the conversion process and preparing the material for the following process, especially if it is waste-derived. Pre-cleaning involves the separation of sand, stones, plastics, metals or any foreign objects that may damage the conversion equipment and contaminate the end product. Cleaning and separation are usually carried out physically using sieving, air separation and metal separation. Such robust technologies are already utilized by the wood pellet industry and carry a high degree of reliability.

Furthermore, another physical pre-treatment involves size reduction. Grinding and milling may also be required since, as discussed, particle size has an impact on the mass and heat transfer which influences reaction heating rate, as well as the general flowability of the material in various reactor designs (Kan et al. 2016). However, size reduction comes at a cost and must be optimized to achieve the highest production efficiencies at the lowest costs. Thermal pre-treatment can also be another requirement in most cases. Depending on feedstock moisture content, a requirement for drying can be unavoidable. Efficient feedstock drying generally promotes a more energy-efficient conversion process. The energy required for drying is usually obtained via re-using gases produced from the pyrolytic process (Kan et al. 2016).

Finally, another uncommon pre-treatment technology that could be employed to influence feedstock properties is de-mineralization. As previously discussed, the inorganic fraction within feedstocks can significantly influence many of the biochar properties discussed. By minimizing the inorganic fraction, various physical and chemical properties can be enhanced; however, this usually hinders biochar yields. De-mineralization technologies are usually adopted prior to fast pyrolysis conversion processes to improve oil yield and characteristics and may include the use of water and chemicals for leaching (Leng et al. 2019b). For the purpose of biochar production, de-mineralization can also be utilized but needs to be carefully assessed from the following aspects, cost, additional wastewater treatment requirement, decreased yield, as well as the general suitability for various applications especially if chemicals are to be utilized.

\section{Biochar: a negative emissions technology}

\section{Carbon sequestration via biochar systems}

Carbon sequestration is the process in which atmospheric carbon is captured and consequently stored in a stable form for extended periods. In relation to all the negative emissions technologies discussed in the literature, biochar has shown to be very promising in many aspects. This includes technical feasibility, potential for scalability, costs, carbon stability and permanence, verification and monitoring as well as application benefits concerning various potential carbon reservoirs (Fawzy et al. 2020). In general, carbon sequestration via biochar production is technically feasible and can be economically viable, especially with the current development of the carbon sink economy. The concept behind biochar is simple and straight forward. Plants capture atmospheric carbon via photosynthesis during plant growth, and as long as the plant continues to live, the carbon is stored within the plant structure. However, as soon as the plant is no longer alive, the natural decay process emits this carbon back to the atmosphere, completing the natural carbon cycle. The production of biochar hacks this carbon cycle by transforming such carbon into a stable form that can persist degradation, preventing emissions to the atmosphere (Qambrani et al. 2017; Brassard et al. 2016).

By combining the photosynthetic route with pyrolytic conversion, an efficient carbon removal system can be designed. At scale, biochar production should ultimately influence the atmospheric carbon balance by reducing atmospheric carbon concentrations. To produce stable biochar that is resistant to decomposition and that can persist in potential reservoirs for hundreds or thousands of years, this requires the appropriate selection of feedstocks and optimization of processing conditions to meet reservoir specific requirements as well as achieve the highest stability possible. Furthermore, this needs to be carried out in the most sustainable approach possible. While biomass waste is a primary focus in terms of urgently restricting potential emissions and promoting the circular economy, the importance and impact of fast-growing dedicated crops should not be undermined, as long as its sustainably cultivated. If dedicated feedstocks are cultivated, the resources utilized in relation to land, water and nutrients should have no direct competition with food production systems. Furthermore, the 
thermochemical conversion process should be carried out in an energy-efficient manner, and the pyrolytic gases or waste heat produced should be re-utilized within the process to prevent emissions as much as possible (EBC 2012). Fossilbased fuels should be eliminated from the production process and minimized as much as possible within cultivation and transportation, if applicable.

Moreover, the final application of the biochar determines its viability as a carbon sink and should be carried out as sustainably as possible, while meeting regulatory and technical requirements. In addition to the sequestration potential of the biochar produced as well as the energy re-utilization potential of the pyrolytic gases, the pyrolytic oils generated can be utilized as a renewable fuel for inhouse energy purposes or to external users, and can also be utilized as a feedstock for renewable chemicals, as shown in Fig. 4. However, the oil will require further upgrading. The sequestration potential for pyrolytic oil has also been presented by Schmidt et al., where such oil can be blended with asphalt binders for road construction or can be stored in geological reservoirs for extended periods. Utilizing the pyrolytic oil in bioplastics and composite materials is another potential sink; however, the persistence of the carbon in such sinks depends on the life cycle of such materials (Schmidt et al. 2019). The utilization of the pyrolytic oil fraction for carbon sequestration purposes depends on the inclusion of such method within the carbon sink economy. Until such method is certified and is remunerated accordingly, the liquid fraction will likely be utilized in applications that maximize its value to the producer. Figure 4 depicts the pyrolytic conversion process showing the various pathways and final reservoirs for the carbon sequestered via biochar, and the various by-product valorization routes.

\section{Potential carbon reservoirs and application benefits}

This section will further explore the potential carbon reservoirs that can provide adequate capacity for long-term storage. Furthermore, the associated application benefits will be presented.

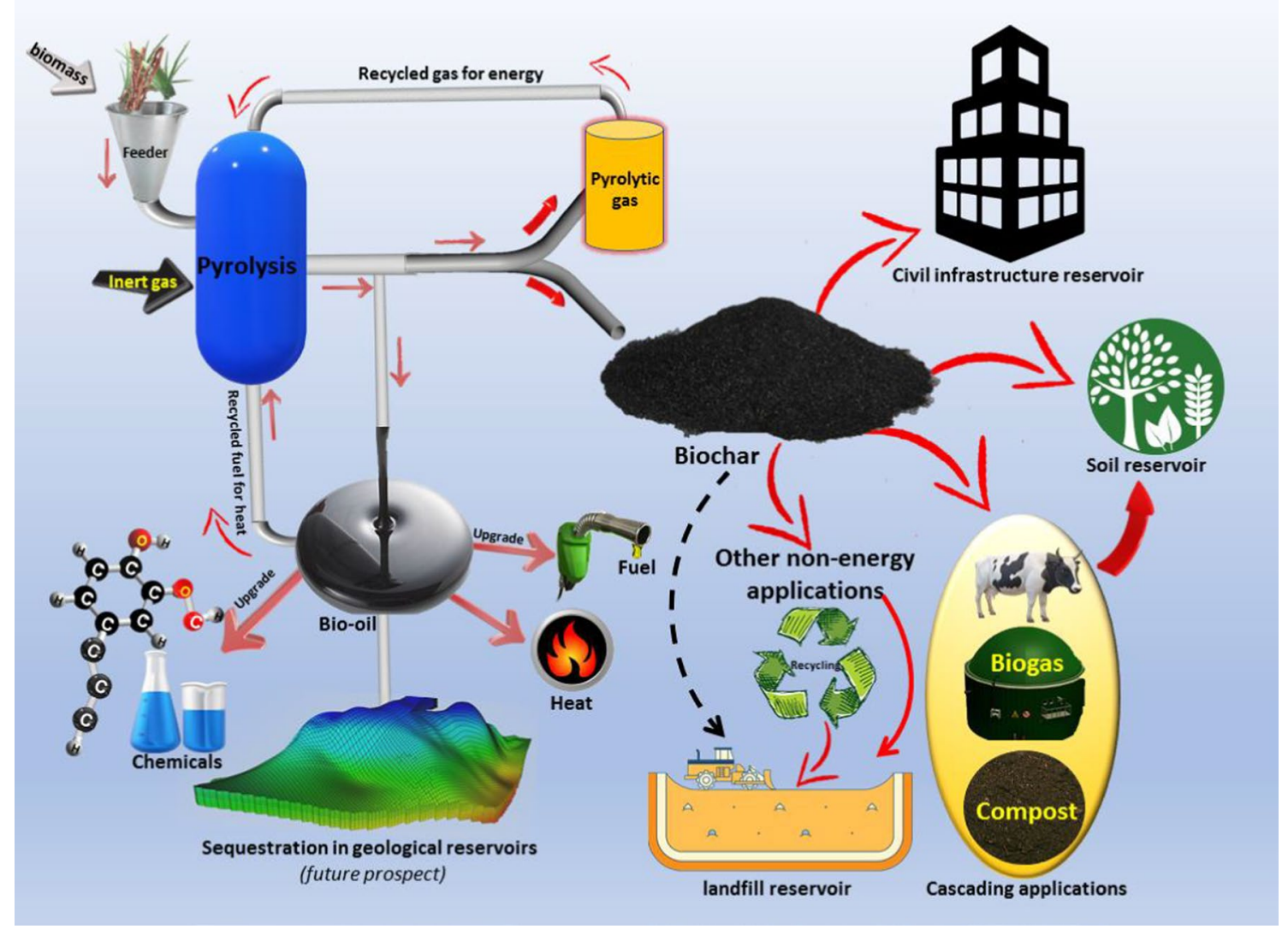

Fig. 4 Pyrolytic conversion process presenting the three main products, biochar, bio-oil and gas. Potential reservoirs for the carbon sequestered in biochar include agricultural and forest soils, civil infrastructure, non-energy related materials and landfills. Prior to the final application within reservoirs, biochar can be utilized in a variety of value-adding applications. Furthermore, valorization routes are presented for the gaseous and liquid fractions 


\section{Agricultural and forest soils}

The long-term storage of biochar in the terrestrial carbon pool via agricultural and forest soils has been extensively investigated within the literature. Soil is perhaps the largest terrestrial carbon reservoir, and the impact of biochar application into soils has gained much academic and commercial interest over the past couple of decades. It has been reported that biochar can be safely stored in soils for centuries if produced under appropriate conditions to achieve carbon stability.

The persistence of carbon in soil has been reported in many studies. Wang et al. investigated biochar stability in soil through a meta-analysis using 24 studies. Based on 128 observations, the researchers meta-analysed the biochar decomposition and computed its mean residence time. The degradation rates varied significantly based on feedstock type, processing conditions, experiment duration and clay content in the soil. The results indicate a mean residence time of 108 days for the labile carbon pool and 556 years for the stable carbon pool, each pool representing 3\% and $97 \%$, respectively. This shows that only a small fraction of biochar is bioavailable and that a significant part contributes to long-term sequestration. When applied to soils, biochar is exposed to biotic and abiotic as well as indirect stresses, which influence the rate of mineralization (Wang et al. 2016). Highly stable carbon should withstand such stresses, and as discussed previously, this comes down to the type of feedstock and processing conditions. In addition to the sequestration potential, biochar has been reported to provide a multitude of agronomic benefits.

In general, it is argued that biochar application can significantly affect soil quality and fertility. Furthermore, improvement in nutrient cycling and an increase in water and nutrient retention have been reported. In theory, the right application of biochar can significantly influence crop productivity and water and nutrient use efficiency. Besides, it has also been reported that biochar application can play an important role in reducing greenhouse gas emissions such as $\mathrm{CO}_{2}, \mathrm{CH}_{4}$, and $\mathrm{N}_{2} \mathrm{O}$ from soils (Fawzy et al. 2020), although the results reported are mixed (Semida et al. 2019; Xiao et al. 2019). Moreover, the sorption capabilities of biochar present an opportunity for it to be utilized for soil remediation (Oni et al. 2019; Xu et al. 2019). The above-mentioned benefits are a result of the impact of biochar on soil physical properties such as porosity and bulk density, soil water dynamics, acidification, the interaction with soil organic matter and inhibition of priming effect, and the stimulation of soil microbial activity and dynamics (Oni et al. 2019; Tenic et al. 2020; Dai et al. 2020). Although the results, in general, show positive effects, there are cases where the application of biochar presented negative results. In general, the results reported in the literature are dependent on the type of biochar, in terms of feedstock and production conditions, amount of biochar applied, type of soil, specific cropping system as well as cultivation management practices (Fawzy et al. 2020; Semida et al. 2019; El-Naggar et al. 2019; Maraseni 2010; Purakayastha et al. 2019).

Biochar can also be utilized in a number of applications prior to the final storage in soils. This cascade effect can maximize biochar value before soil application. The literature reports the use of biochar within the composting production process, where process and product benefits can be realized in terms of lower process emissions during production and enhanced end-product quality (Kuppusamy 2019). Furthermore, biochar use as an animal feed additive has been investigated in the literature showing many positive results. Documented results include improved animal growth, ability to resist disease, reduction of methane production and the removal of toxins (Man et al. 2021). Furthermore, Schmidt highlighted several other animal farming applications, namely as a silage agent, litter additive, slurry treatment, manure composting and the treatment of water from fish farming (Schmidt 2013). Water remediation such as nitrogen and phosphorus removal from aquatic systems can also be an interesting application where the resulting nutrient-loaded biochar can be further applied to soils (Zhang et al. 2020). The role of biochar in anaerobic digestion has also been investigated in the literature, showing enhanced gas yield (Schmidt 2013; Meyer-Kohlstock et al. 2016; Wambugu et al. 2019). However, while optimizing biochar production to meet application-specific requirements, carbon stability remains the prominent parameter for biochar to serve its sequestration purpose once it is finally applied to soil.

Finally, in relation to risks associated with large-scale deployment of biochar in soils, albedo effect has been reported. The decrease in surface reflectivity due to high application rates of biochar is argued to potentially increase soil temperature, reducing the benefits achieved through this sequestration route (Fawzy et al. 2020; Fuss et al. 2018). The scientific community should further look into this topic, where application rate thresholds can be devised.

\section{Civil infrastructure}

Long-term storage can also be achieved within civil infrastructure such as asphalt roads, pavements, buildings and various structural applications. The potential of biochar to be utilized as a building material has been investigated extensively within the literature. Three main properties have been highlighted that suggest the suitability of biochar to be introduced into construction; its high chemical stability, low thermal conductivity and low flammability.

Chemical stability is important to ensure that no harmful chemical reactions occur when biochar is mixed with other components in an asphalt or concrete mix. In general, 
concrete is known to suffer chemical attacks which ultimately reduce its durability. Furthermore, asphalt suffers degradation due to oxidation, which profoundly affects the durability and stability of roads and pavements. The chemical stability of biochar once mixed with concrete or asphalt restricts the possibility of such detrimental chemical reactions and ensures long-term durability. Furthermore, the low thermal conductivity enhances insulation potential for buildings and structures. Porosity and specifically pore size distribution is the main factor contributing to this property. Finally, low flammability is an extremely important parameter from a safety point of view (Gupta and Kua 2017).

Moreover, biochar's water holding capacity has shown to offer adequate hydration capabilities in cementitious admixtures, promoting enhanced internal curing. This leads to improvements in durability, shrinkage, cracking and mechanical properties (Akinyemi and Adesina 2020). In addition, the literature generally reports structural benefits in association with biochar incorporation into cement-based composites, where improvements in mechanical properties, such as compressive and flexural strength as well as ductility and general toughness, are documented (Choi et al. 2012b; Restuccia and Ferro 2016; Khushnood et al. 2016; Ahmad et al. 2015). Reduction in setting times as well as enhanced durability have also been reported (Akinyemi and Adesina 2020).

Furthermore, various benefits associated with using biochar as a modifying additive with bitumen for asphalt applications have been reported which include, improvement of the mechanical and rheological properties of the material, improvement in viscosity and rutting resistance of the asphalt, reduction in temperature susceptibility, and reduction of oxidation and ageing of asphalt (Ahmad et al. 2015; Zhao et al. 2014a, b; Abu-Lebdeh 2014). The potential for biochar to be incorporated within the built environment for long-term storage promotes the concept that civil infrastructure is a solid carbon reservoir. In addition to long-term carbon storage, the use of biochar as a supplementary cementitious material, by partially replacing cement in admixtures, provides further emission reduction benefits, since cement production is a carbon-intensive process. However, it is important to note that the type of feedstock, processing conditions and application rates play a critical role in allowing biochar to be introduced into such applications as well as provide the above-mentioned benefits.

\section{Other applications}

Biochar can be used in other applications such as industrial materials, e.g. plastics and electronics, wastewater treatment, stormwater treatment and many other applications (Schmidt 2013). Biochar can serve as a carbon sink in these applications as long as it is not related to energy production. In addition, these products must not be exposed to thermal degradation or oxidation throughout or at the end of their service life (Schmidt et al. 2019). The reversible nature of some of these products weakens the recognition of such applications as carbon sinks. However, this is not the case for applications where the final product can be adequately disposed of and easily monitored. If materials are recycled, the sequestered carbon remains in the terrestrial pool for as long as the material is not subjected to thermal degradation or oxidation. Once the material reaches the end of its life cycle, it can be safely disposed of in landfills, where the sequestered carbon can be stored for extended periods. Landfill sequestration is also a valid route for non-recyclable or contaminated biochar materials.

Furthermore, another basic concept is the direct burial of biochar in landfills, as a long-term storage reservoir. The concept of applying biochar to the soil cover of landfills for the mitigation of methane emissions has been investigated in the literature, showing positive results (Wu et al. 2020). However, the idea of direct burial of biochar in landfills mainly revolves around the long-term safe storage of sequestered carbon. Additional benefits of this approach should be further investigated, if any. This approach can make sense only if it is commercially viable to do so. This could be an option for biochar that is only designed for carbon sequestration purposes, e.g. the biochar exhibits carbon stability but other physicochemical properties are not sufficient to meet regulatory or value-added application specific requirements.

\section{An emerging carbon removal economy}

While the world is familiar with conventional mitigation efforts, and the concept of reducing emissions, the need for further atmospheric carbon removal is profound. To reach the targets required by the Paris agreement, emission reductions must be accompanied with further atmospheric carbon removal efforts. Thus far, the carbon economy has been built on regulatory mechanisms such as carbon taxation and mandatory and voluntary market-based mechanisms such as emission trading systems. The integration of negative emissions trading within the carbon economy is critical to the success of carbon removal as a climate change combat tool moving forward. To succeed, carbon removal must be viewed and treated as a critical service to achieve the required reduction in global temperatures, and therefore must be remunerated accordingly. For the carbon removal market to develop, various frameworks and standards must be developed to allow market participants to offer their carbon removal services in a quantifiable and environmentally sustainable way. Methodologies must be developed and globally standardized for each technology to adequately quantify, monitor, facilitate and govern carbon removal. 
At the moment, carbon removal efforts are mainly promoted via the voluntary market, where various carbon removal service models are observed. In relation to carbon removal through afforestation and reforestation efforts, this is perhaps the oldest technique adopted globally and has already been integrated within climate policies. Tradeable credits, termed removal units, have been introduced through the Kyoto protocol since the late $90 \mathrm{~s}$. Forestation projects have also been integrated within national regulations, and in 2008, the United Nations introduced a voluntary program called reducing emissions from deforestation and forest degradation (REDD +). However, despite all these efforts, sequestration through forestation only accounted for $0.5 \%$ of the total volume of tradeable carbon in 2013 (Fawzy et al. 2020; Gren and Aklilu 2016). In addition to these measures, forest-based project developers are reaching out directly to the public to secure sufficient financing to grow and upgrade forests, while providing the general public with a tool to offset personal and business-related emissions. Financing is either raised through donations via non-profit organizations, such as one tree planted in the USA (OneTreePlanted 2021) or woodland trust in the UK (WoodlandTrust 2021), or through carbon removal service fees by commercial developers. Forest carbon, a commercial developer, offers one-off purchases and monthly subscriptions for carbon removal services through the creation of new woodlands in the UK. The company offers three tiers of membership, offering single purchases valued at an average price of 10.8 GBP per ton $\mathrm{CO}_{2}$, as well as packages for households and small businesses offered at varying rates per ton of $\mathrm{CO}_{2}$ on a monthly subscription (ForestCarbon 2021).

Direct air carbon capture and storage is an interesting technology that comes with its merits and challenges. One of the prominent leaders in this field is Swiss company climeworks, which have commercialized their technology and are offering their carbon removal services directly to business and the general public. Similar to forest-based project developers, climeworks are offering their services on a monthly subscription basis. The monthly service rates are 6,9 and 44 GBP for an annual removal of 85,255 and $600 \mathrm{~kg} \mathrm{CO}_{2}$, respectively, which translates to a range of 847-895 GBP per ton $\mathrm{CO}_{2}$. Furthermore, the company offers a flexible monthly payment plan from 1 to 2000 GBP for the annual removal of 14-27,618 $\mathrm{kg} \mathrm{CO}_{2}$, translating to approximately $869 \mathrm{GBP}$ per ton $\mathrm{CO}_{2}$ (Climeworks 2021). Prices seem high currently, however, as the company scales, prices should drop in the near future. Furthermore, one would assume that large purchases can also be carried out directly with the company at agreed rates. Although the concept behind offering direct carbon removal services to the market is interesting, the lack of monitoring and control through an independent body deems this approach questionable. There needs to be some form of governance, monitoring and verification. Carbon removal through direct air carbon capture and storage is at a very early stage, and as more participants enter the market, the creation of a governing body will probably be required.

In 2019 and 2020, the world experienced the creation of a number of voluntary carbon removal platforms, pioneered by three different organizations. The nori carbon removal marketplace in the USA specializes in soil carbon sequestration. They developed standards and a methodology for quantification, monitoring and verification and are currently connecting farmers who have been independently audited with entities and individuals interested in carbon removal. The Nori platform is based on block-chain technology and offers a high degree of transparency. Currently, carbon removal via soil carbon sequestration is valued at $10.8 \mathrm{GBP}$ per ton $\mathrm{CO}_{2}$, in addition to a $15 \%$ transaction fee (Nori 2021).

Puro, a Finnish start-up, is probably the first carbon removal marketplace launched. The company has developed methodologies and verification processes for three carbon removal technologies, biochar, carbonated building elements and wooden building elements. To certify potential carbon removal suppliers, each potential supplier undergoes an extensive verification process. For biochar producers, the company requires the produced biochar and the production process to be certified by either the European biochar certificate or the international biochar initiative in the USA. Otherwise, a certified life cycle assessment needs to be presented. Furthermore, a production facility audit is also carried out by an independent auditor. The methodologies provide rules for quantification of carbon removal as well as verification and continuous monitoring.

At the moment, puro hosts ten carbon removal suppliers, six of which are biochar producers and the remaining offer sequestration through wooden building elements. Currently, the carbon removal service fees through biochar range from 52 to 131 GBP per ton $\mathrm{CO}_{2}$, while through wooden building elements service fees range from 17 to 23 GBP per ton $\mathrm{CO}_{2}$. These prices already include puro's service fees and are inclusive of value added tax. This marketplace operates on an auction basis, whereby the carbon removal certificates generated by the suppliers are auctioned to businesses and individuals. Furthermore, direct purchases can be made through Puro's website, and pre-purchase agreements can also be arranged between suppliers and sellers (Puro.earth 2021a). One of the main advantages of this platform is the opportunity for suppliers to decouple the carbon removal service from the sale of the actual product, providing two revenue streams. Finally, the fact that an independent body can verify and monitor the whole process adds to the complete system's credibility.

Carbon future is another marketplace provider based in Germany, which specializes in biochar as a carbon removal method. This block-chain based platform integrates the full supply chain from production to final application of the 
biochar to ensure carbon removal authenticity. The platform does this by issuing two documents, the production certificate and a coupon that is created once the biochar has been finally applied into a stable reservoir; it is only at this point that the system issues a carbon removal certificate or credit that is tradeable. Furthermore, similar to puro, participation within this ecosystem requires the biochar and the production process to be certified by the European biochar certificate or the international biochar initiative. The current carbon removal credits are offered at $104 \mathrm{GBP}$ per ton $\mathrm{CO}_{2}$, which includes 19\% value added tax (CarbonFuture 2021).

While most of the current efforts are promoted through voluntary markets, the integration of such methods into climate policies and official trading systems would further enhance market development. Carbon pricing, integration into policy, financial support and accessibility for project developers, the streamlining and standardization of digital platforms, and further technology research and development, are required to push this marketplace forward. The following section will further explore certification and eligibility requirements as well as methodologies for carbon sequestration quantification via biochar within this new marketplace.

\section{Carbon removal marketplace participation requirements}

Biochar and production process certification are current prerequisites for the eligibility of biochar producers to become participants within the emerging carbon removal marketplace. Currently, only two marketplace providers facilitate carbon removal via biochar, puro and carbon future. The carbon future marketplace requires that the biochar and production process must be certified by the European biochar certificate or similar certification by another entity. Puro marketplace also requires the production process to be certified by the European biochar certificate or a similar certification body; however, if the production process is not certified an official life cycle assessment must be prepared and presented. In general, as stipulated in their methodology, most of the requirements for participation in the puro marketplace are in line with the European biochar certificate requirements, with a further requirement for an additional independent facility audit. Each marketplace has its own carbon removal quantification protocol, based on the activity boundaries and uncertainty buffers defined by each marketplace provider (Puro.earth 2021b; CarbonFuture 2020). Details of such protocols can be accessed through each platform's detailed methodologies. The following subsection will introduce the certification requirements stipulated by the European biochar certificate and the international biochar initiative as they are accredited certification entities. In addition to facilitating access to the carbon removal market, the certification process is a value-adding activity as it adds authenticity and credibility to the biochar producer for marketing the physical biochar.

\section{Certification}

The European biochar certificate, also referred to as EBC, is a well-recognized voluntary body setting standards for sustainable biochar production in Europe. The guidelines developed provide all necessary requirements and details for the successful certification of biochar producers. In general, four classes of biochar are promoted which are mainly based on application, EBC-Feed, EBC-AgroBio, EBC-Agro and EBC-Material. Each of these classes carries their own requirements in terms of meeting application-specific regulations. The certification process includes an overall assessment on feedstock eligibility, production process eligibility and requirements, sampling, labelling and quality management procedures, health and safety regulations, and most importantly biochar properties. Properties must meet regulatory thresholds for each of the defined classes. Certified analytical techniques are also provided within the guidelines. Each area of assessment is covered in detail within the guidelines. Table 3 presents a summary of the declaration requirements and thresholds for the specified biochar properties for each of the application classes defined (EBC 2012). The European biochar certificate has also developed standards for the certification of biochar-based carbon sinks, including detailed guidelines for quantifying carbon sequestration potential (EBC 2020). The carbon future platform mainly adheres to the carbon sink certification protocol developed by the European biochar certificate, while puro has customized their own protocol, yet it is in line with the general requirements. The carbon sink certification guidelines cover the following points (EBC 2020).

1. A definition of and calculation of carbon sink potential: The carbon sink potential of biochar is calculated by determining the carbon content of the biochar less all emissions realized from the provision of feedstock, the storage of biomass, the pyrolysis process and a safety margin. The calculation accounts for the full carbon footprint of the biochar from the feedstock source to biochar that is ready to leave the production plant.

2. A definition of the carbon neutrality of feedstocks utilized: Only carbon-neutral feedstocks are allowed to be used for the certification of biochar-based carbon sinks. Residual biomass is classified as carbon neutral. Furthermore, dedicated crops are permitted if there is no reduction in the total carbon stock of the system during their growth and harvest, and any carbon emissions resulting in the cultivation practices are accounted for. Biomass obtained via the destruction of forests and other natural carbon sinks are not permitted. 


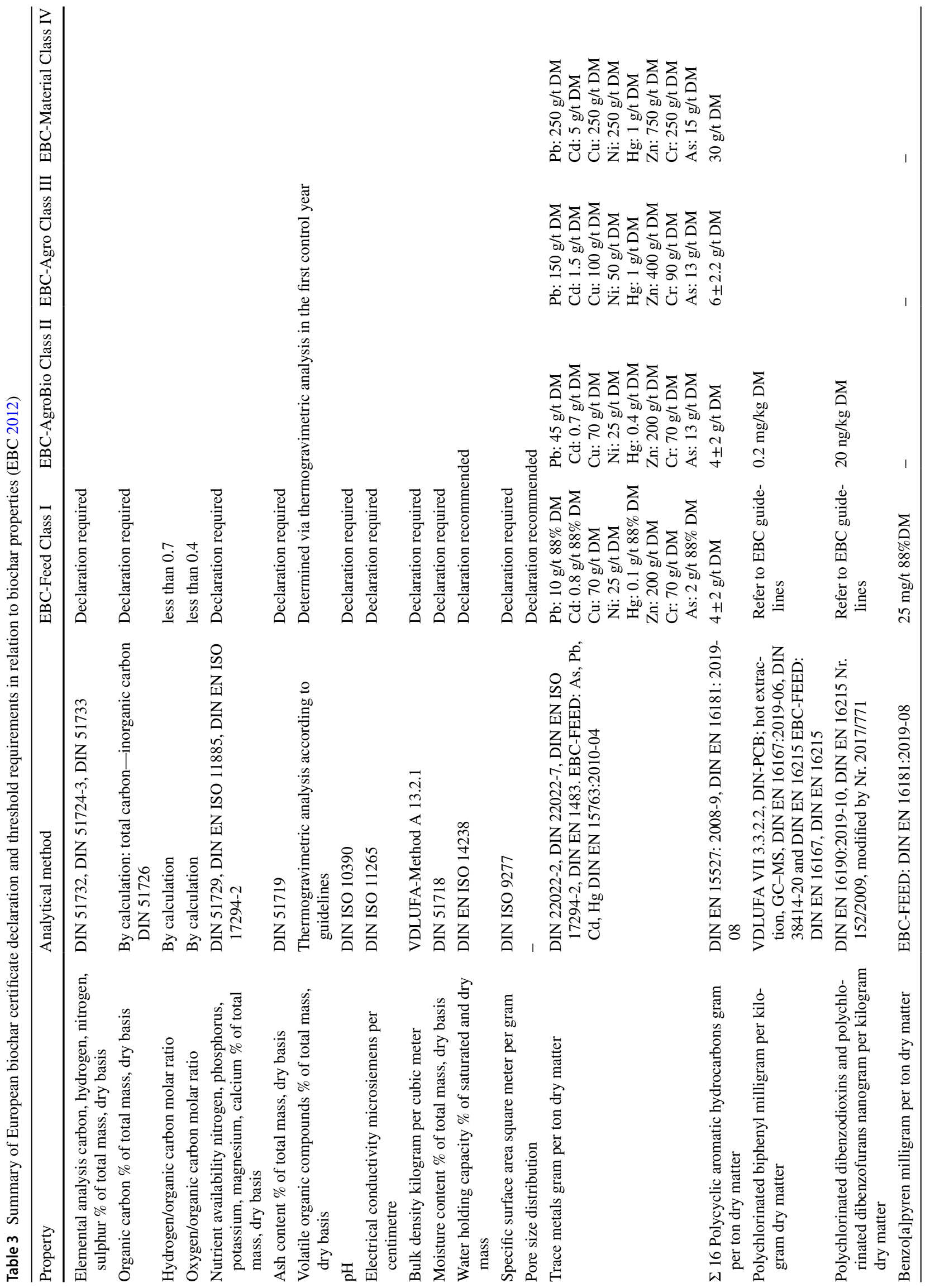


3. Eligibility of feedstocks and a discussion of each biomass category and calculation of carbon expenditure for the provision of such biomass: The guidelines specify six eligible biomass categories, namely (1) agricultural biomass, (2) organic residues from food processing, (3) wood from landscape conservation, short rotation plantations, forest gardens, urban areas, and arable forestry, (4) biomass from forest management, (5) wood waste and (6) other biogenic residues. Carbon expenditure calculations for feedstock provision are presented within the guidelines.

4. Storage of biomass and the consideration for the resulting emissions and provisions made within the carbon removal quantification protocol: Emissions resulting from the storage of wet biomass, if any, need to be accounted for in calculating the carbon sink potential. Recommendations for adequate storage of biomass are also provided to minimize potential emissions.

5. Deductions for emissions related to biochar production and calculation of carbon expenditure for the production process: Emissions related to the transportation of the biomass to the plant, any production-related emissions, as well as transportation of the end product to the factory gates, need to be accounted for. Provisions for the use of electricity and fuel consumption in each of the production phases are made. Any source of external energy utilized in the production process is accounted for. Furthermore, any methane emissions realized from the pyrolytic process needs to be adequately deducted. The guidelines provide an example for the calculation of production process related carbon expenditure.

6. Mandatory data requirements for carbon sink certification: This mainly includes information in relation to the on-site measurement techniques required.

7. Discussion and quantification regarding the margin of safety for carbon expenditures: A margin of safety of $10 \%$ of the total emissions realized is deducted in the carbon footprint calculation as a provision for indirect emissions that are not accounted for.

8. The separation between carbon sink certification for biochar produced and climate-neutral energy that is produced in parallel, setting boundaries for certification coverage.

9. A discussion on the use and trade of the biochar-based carbon sinks by platforms and brokers: Any emissions realized after the biochar leaves the processing plant should be accounted for and deducted from the total carbon sink potential of the biochar. For this reason, a tracking system is required to record all the carbon losses incurred from the factory to the final end-use application where the biochar is applied to soil or incorporated in long-term stable materials. The guidelines provide further details in relation to provisions made for the degradation of the biochar in various applications. In soil and related cascaded applications, based on the $\mathrm{H} / \mathrm{C}_{\text {org }}$ ratio an annual degradation rate of $0.3 \%$ may be required to account for potential mineralization. This would not be required for material-based applications. However, appropriate monitoring, verification and reporting are required. The European biochar certificate has also developed an accreditation protocol for trading platforms and carbon sink traders to ensure that tracking systems adhere to the required criteria.

The international biochar initiative, also referred to as IBI, is a voluntary biochar certification and standard-setting entity operating in the USA. Similar to the European biochar certificate, the international biochar initiative has developed standards and procedures for the successful certification of biochar producers. The standards cover feedstock eligibility, declaration of biochar properties and regulatory thresholds, general protocols and restrictions, as well as recommendations on best management practices for production, handling and storage. Unlike the European biochar certificate, the international biochar initiative does not prescribe production process requirements for certification. This means that at the moment, the European biochar certificate is the only entity offering production process certification. However, biochar certification by the international biochar initiative is acknowledged by the marketplaces (CarbonFuture 2020). Product property testing is carried out in three categories, category a: basic utility properties, category b: toxicant assessment and category c: advanced analysis and soil enhancement properties. The first two categories are mandatory for certification, while the declaration of the third is optional. A summary of the product property criteria and analytical methods used is presented in Table 4 (IBI 2015; Rajkovich et al. 2011; Van Zwieten et al. 2010; Rayment and Higginson 1992; Enders and Lehmann 2012; Wang et al. 2012; Agriculture 2001).

\section{Conclusion}

Carbon removal is becoming an inevitable strategy for the mitigation of climate change since emission reduction alone is deemed insufficient in tackling the current state of climate emergency. Currently, a carbon removal marketplace is emerging, and the next few years will shape the future of this industry. Biochar production is a valid and promising carbon removal technique that relies on a hybrid biogenic and technological framework. As discussed, large-scale deployment of biochar production would significantly contribute towards reducing atmospheric carbon concentrations and ultimately support in reducing global temperatures. The production of 


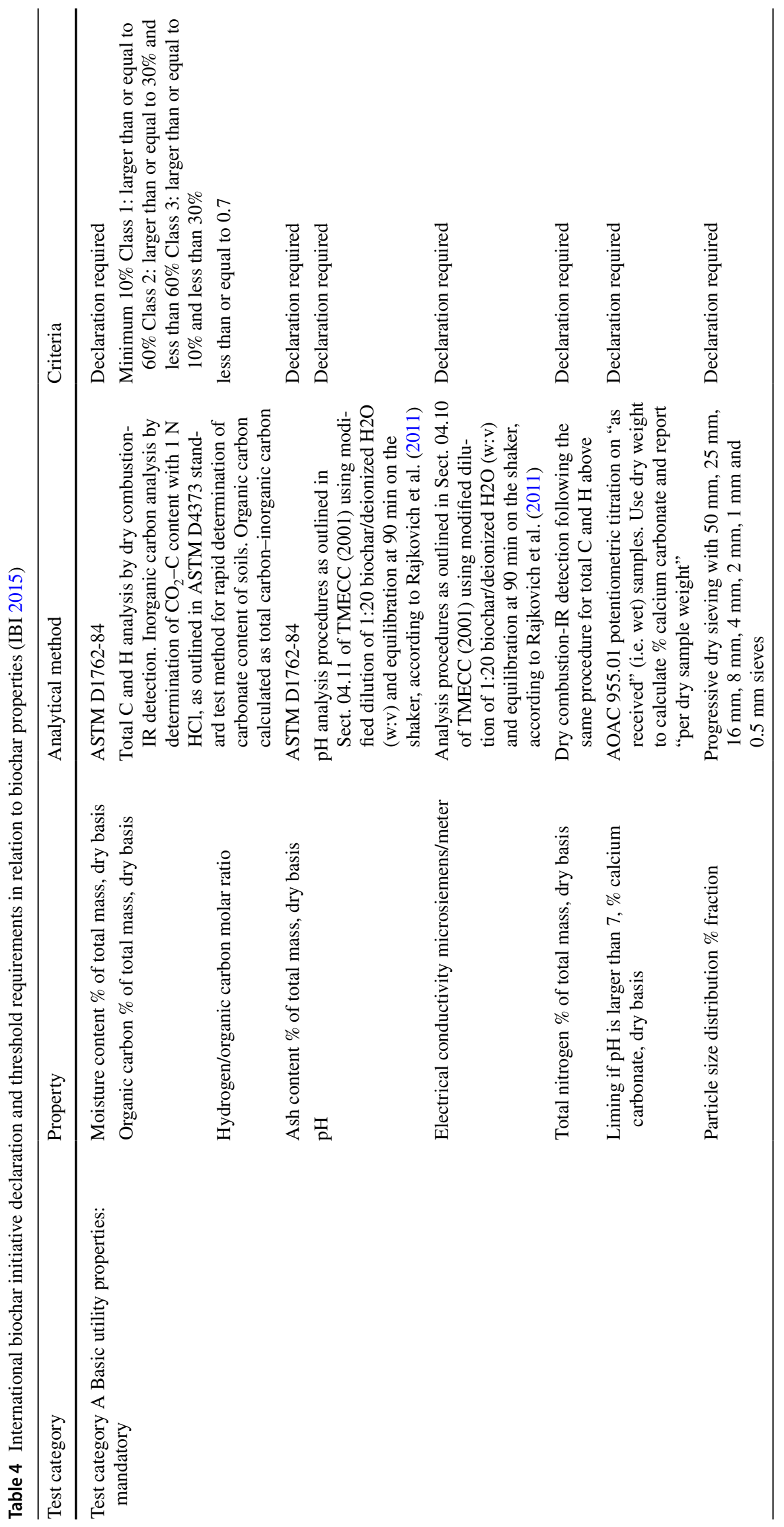




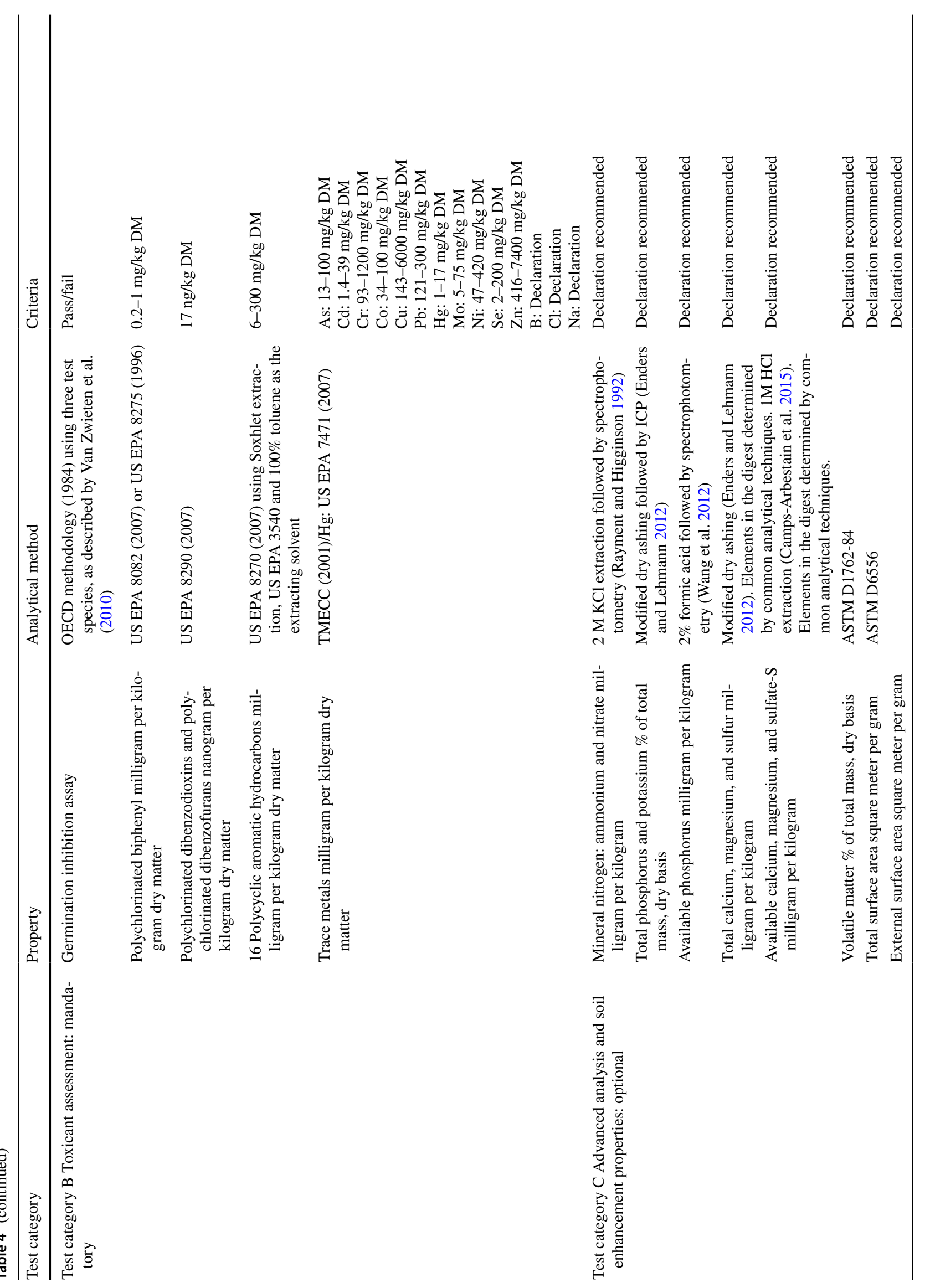


biochar has, thus far, been focused on agronomic as well as a variety of other applications, and extensive research is being carried out to develop more useful applications within various industrial and agricultural sectors. With the current climate-related crisis and the dire need to develop a carbon removal market, biochar production geared towards atmospheric carbon removal is an emerging topic.

This literature review explored areas that can guide in the design and development of an industrial biochar production system geared towards atmospheric carbon removal. The investigation covered potential feedstocks, feedstock analytical techniques, potential production technologies, biochar properties with a special focus on carbon stability, the impact of processing configurations on production yield and biochar properties, and presented potential by-product valorization routes. Furthermore, the article discussed the concept of biochar as a negative emissions technology and introduced potential carbon reservoirs and a variety of valueadding applications. The investigation also covered the current status of the emerging carbon removal economy and discussed participation requirements. It is important to note that the continuous development of the carbon removal market and the integration of negative emissions trading within policy frameworks and official markets will be imperative for the continued success of this new industry.

As discussed in the review, biochar production requires extensive knowledge and understanding to achieve an end product that meets application-specific requirements and achieve ultimate stability for carbon sequestration purposes. This requires on-going product-process optimization to achieve optimal processing conditions based on feedstock type and end application. Furthermore, for the successful development of industrial-scale projects, techno-economic investigations are of extreme importance. Therefore, in moving forward, it is suggested that further research should focus on system design, process optimization and technoeconomic feasibility assessments of biochar-based carbon removal projects. With the emergence of the new carbon removal economy, this line of applied research would be of great value for entrepreneurs, investors, financiers, policymakers and various potential market participants to better understand the dynamics behind commercial carbon removal projects via biochar production.

Acknowledgements Authors would like to acknowledge the support given by the EPSRC project "Advancing Creative Circular Economies for Plastics via Technological-Social Transitions" (ACCEPT Transitions, EP/S025545/1). The authors wish to acknowledge the support of The Bryden Centre project (Project ID VA5048) which was awarded by The European Union's INTERREG VA Programme, managed by the Special EU Programmes Body (SEUPB), with match funding provided by the Department for the Economy in Northern Ireland and the Department of Business, Enterprise and Innovation in the Republic of Ireland.
Open Access This article is licensed under a Creative Commons Attribution 4.0 International License, which permits use, sharing, adaptation, distribution and reproduction in any medium or format, as long as you give appropriate credit to the original author(s) and the source, provide a link to the Creative Commons licence, and indicate if changes were made. The images or other third party material in this article are included in the article's Creative Commons licence, unless indicated otherwise in a credit line to the material. If material is not included in the article's Creative Commons licence and your intended use is not permitted by statutory regulation or exceeds the permitted use, you will need to obtain permission directly from the copyright holder. To view a copy of this licence, visit http://creativecommons.org/licenses/by/4.0/.

\section{References}

Abdel Maksoud MIA et al (2020) Advanced materials and technologies for supercapacitors used in energy conversion and storage: a review. Environ Chem Lett 19:375-439. https://doi.org/10.1007/ s10311-020-01075-w

Abu-Lebdeh T (2014) Enhancing asphalt rheological behavior and aging susceptibility using bio-char and nano-clay. Am J Eng Appl Sci 7:66-76

Acar S, Ayanoglu A (2012) Determination of higher heating values (HHVs) of biomass fuels. Energy Educ Sci Technol A Energy Sci Res 28:749-758

Afolabi OOD et al (2020) Optimisation and characterisation of hydrochar production from spent coffee grounds by hydrothermal carbonisation. Renew Energy 147:1380-1391. https://doi. org/10.1016/j.renene.2019.09.098

Agriculture, U. C. C. a. U. D. o. (2001) Test methods for the examination of composting and compost. (TMECC) Thompson W.H. (ed.) http://compostingcouncil.org/tmecc/. Accessed 29 Jan 2021

Ahmad S et al (2015) High performance self-consolidating cementitious composites by using micro carbonized bamboo particles. Mater Des 76:223-229. https://doi.org/10.1016/j.matde s.2015.03.048

Akhil D et al (2021) Production, characterization, activation and environmental applications of engineered biochar: a review. Environ Chem Lett. https://doi.org/10.1007/s10311-020-01167-7

Akinyemi BA, Adesina A (2020) Recent advancements in the use of biochar for cementitious applications: a review. J Build Eng 32:101705. https://doi.org/10.1016/j.jobe.2020.101705

Akor CI et al (2021) Thermokinetic study of residual solid digestate from anaerobic digestion. Chem Eng J 406:127039. https://doi. org/10.1016/j.cej.2020.127039

Angin D (2013) Effect of pyrolysis temperature and heating rate on biochar obtained from pyrolysis of safflower seed press cake. Bioresour Technol 128:593-597. https://doi.org/10.1016/j.biort ech.2012.10.150

Antal MJ, Grønli M (2003) The art, science, and technology of charcoal production. Ind Eng Chem Res 42:1619-1640. https://doi. org/10.1021/ie0207919

Antal MJ et al (1996) High-yield biomass charcoal. Energy Fuels 10:652-658. https://doi.org/10.1021/ef9501859

Antal MJ et al (2003) Flash carbonization of biomass. Ind Eng Chem Res 42:3690-3699. https://doi.org/10.1021/ie0301839

Aysu T, Küçük MM (2014) Biomass pyrolysis in a fixed-bed reactor: effects of pyrolysis parameters on product yields and characterization of products. Energy 64:1002-1025. https://doi. org/10.1016/j.energy.2013.11.053 
Balajii M, Niju S (2019) Biochar-derived heterogeneous catalysts for biodiesel production. Environ Chem Lett 17:1447-1469. https:// doi.org/10.1007/s10311-019-00885-x

Banik C et al (2018) Impact of pyrolysis temperature and feedstock on surface charge and functional group chemistry of biochars. J Environ Qual. https://doi.org/10.2134/jeq2017.11.0432

Basu P (2013) Chapter 13-analytical techniques. In: Basu P (ed) Biomass gasification, pyrolysis and torrefaction, 2nd ed. Academic Press, Boston, pp 439-455. https://doi.org/10.1016/B978-0-12396488-5.00013-7

Batista EMCC et al (2018) Effect of surface and porosity of biochar on water holding capacity aiming indirectly at preservation of the Amazon biome. Sci Rep 8:10677. https://doi.org/10.1038/ s41598-018-28794-z

Blackadder W, Rensfelt E (1985) A pressurized thermo balance for pyrolysis and gasification studies of biomass, wood and peat. In: Overend RP et al (eds) Fundamentals of thermochemical biomass conversion. Springer, Dordrecht, pp 747-759. https://doi. org/10.1007/978-94-009-4932-4_39

Bonilla J et al (2019) Kinetic triplet of Colombian sawmill wastes using thermogravimetric analysis. Heliyon 5:e02723. https://doi. org/10.1016/j.heliyon.2019.e02723

Brassard P et al (2016) Soil biochar amendment as a climate change mitigation tool: key parameters and mechanisms involved. J Environ Manag 181:484-497. https://doi.org/10.1016/j.jenvm an.2016.06.063

Brassard P et al (2017) Pyrolysis in auger reactors for biochar and bio-oil production: a review. Biosyst Eng 161:80-92. https://doi. org/10.1016/j.biosystemseng.2017.06.020

Brown RA et al (2006) Production and characterization of synthetic wood chars for use as surrogates for natural sorbents. Organ Geochem 37:321-333. https://doi.org/10.1016/j.orggeochem .2005.10.008

Bruun EW et al (2012) Effects of slow and fast pyrolysis biochar on soil $\mathrm{C}$ and $\mathrm{N}$ turnover dynamics. Soil Biol Biochem 46:73-79. https://doi.org/10.1016/j.soilbio.2011.11.019

Buss W et al (2016) Strategies for producing biochars with minimum PAH contamination. J Anal Appl Pyrolys 119:24-30. https://doi. org/10.1016/j.jaap.2016.04.001

Cai $\mathbf{J}$ et al (2017) Review of physicochemical properties and analytical characterization of lignocellulosic biomass. Renew Sustain Energy Rev 76:309-322. https://doi.org/10.1016/j. rser.2017.03.072

Calvelo Pereira R et al (2011) Contribution to characterisation of biochar to estimate the labile fraction of carbon. Organ Geochem 42:1331-1342. https://doi.org/10.1016/j.orggeochem .2011.09.002

Camps-Arbestain M et al (2015) A biochar classification system and associated test methods. In: Lehmann J, Joseph S (eds) Biochar for environmental management: science, technology and implementation. Routledge, New York, pp 165-194

CarbonFuture (2020) Playbook for biochar producer and wholesaler, Version 2.0. https://www.carbonfuture.earth/resources. Accessed 29 Jan 2021

CarbonFuture (2021) https://www.carbonfuture.earth Accessed 27 Jan 2021

CE B (2012) Biochar characterization and engineering. In: Doctoral dissertation. Iowa State University, pp 55-57

Cetin E et al (2004) Influence of pyrolysis conditions on the structure and gasification reactivity of biomass chars. Fuel 83:2139-2150. https://doi.org/10.1016/j.fuel.2004.05.008

Chen B et al (2008) Transitional adsorption and partition of nonpolar and polar aromatic contaminants by biochars of pine needles with different pyrolytic temperatures. Environ Sci Technol 42:51375143. https://doi.org/10.1021/es8002684
Chen W et al (2019) Past, present, and future of biochar. Biochar 1:7587. https://doi.org/10.1007/s42773-019-00008-3

Choi HS et al (2012a) Fast pyrolysis characteristics of lignocellulosic biomass with varying reaction conditions. Renew Energy 42:131-135. https://doi.org/10.1016/j.renene.2011.08.049

Choi W et al (2012b) Mechanical properties of mortar containing biochar from pyrolysis. J Korea Inst Struct Maintenan Inspect 16, 67-74. https://doi.org/10.11112/jksmi.2012.16.3.067

Chun Y et al (2004) Compositions and sorptive properties of crop residue-derived chars. Environ Sci Technol 38:4649-4655. https ://doi.org/10.1021/es035034w

Climeworks (2021) https://www.climeworks.com. Accessed 27 Jan 2021

Crombie K et al (2013) The effect of pyrolysis conditions on biochar stability as determined by three methods. GCB Bioenergy 5:122131. https://doi.org/10.1111/gcbb.12030

Cross A, Sohi SP (2013) A method for screening the relative longterm stability of biochar. GCB Bioenergy 5:215-220. https://doi. org/10.1111/gcbb. 12035

Dai Y et al (2020) Combined effects of biochar properties and soil conditions on plant growth: a meta-analysis. Sci Total Environ 713:136635. https://doi.org/10.1016/j.scitotenv.2020.136635

De la Rosa JM et al (2019) Effect of pyrolysis conditions on the total contents of polycyclic aromatic hydrocarbons in biochars produced from organic residues: assessment of their hazard potential. Sci Total Environ 667:578-585. https://doi.org/10.1016/j. scitotenv.2019.02.421

de Resende MF et al (2018) Polycyclic aromatic hydrocarbons in biochar amended soils: long-term experiments in Brazilian tropical areas. Chemosphere 200:641-648. https://doi. org/10.1016/j.chemosphere.2018.02.139

Demiral İ, Ayan EA (2011) Pyrolysis of grape bagasse: effect of pyrolysis conditions on the product yields and characterization of the liquid product. Bioresour Technol 102:3946-3951. https ://doi.org/10.1016/j.biortech.2010.11.077

Demirbas A (2004) Effects of temperature and particle size on bio-char yield from pyrolysis of agricultural residues. J Anal Appl Pyrolys 72:243-248. https://doi.org/10.1016/j. jaap.2004.07.003

Demirbas A (2009) Pyrolysis mechanisms of biomass materials. Energy Sources A Recov Util Environ Eff 31:1186-1193. https ://doi.org/10.1080/15567030801952268

Dhyani V, Bhaskar T (2018) A comprehensive review on the pyrolysis of lignocellulosic biomass. Renew Energy 129:695-716. https:// doi.org/10.1016/j.renene.2017.04.035

Downie A et al (2009) Physical properties of biochar. Biochar Environ Manag Sci Technol 4:13-32

EBC (2012) European Biochar Certificate-guidelines for a sustainable production of biochar. European Biochar Foundation (EBC), Arbaz, Switzerland. http://European-biochar.org. Version 9.2E of 2nd December 2020

EBC (2020) Certification of the carbon sink potential of biochar, Ithaka Institute, Arbaz, Switzerland. http://European-biochar.org. Version $1.0 \mathrm{E}$ of 1 st June 2021

El-Naggar A et al (2019) Biochar application to low fertility soils: a review of current status, and future prospects. Geoderma 337:536-554. https://doi.org/10.1016/j.geoderma.2018.09.034

Encinar JM et al (2000) Fixed-bed pyrolysis of Cynara cardunculus L. Product yields and compositions. Fuel Process Technol 68:209222. https://doi.org/10.1016/S0378-3820(00)00125-9

Enders A, Lehmann J (2012) Comparison of wet-digestion and dryashing methods for total elemental analysis of biochar. Commun Soil Sci Plant Anal 43:1042-1052. https://doi.org/10.1080/00103 624.2012 .656167

Ertaş M, Hakkı Alma M (2010) Pyrolysis of laurel (Laurus nobilis L.) extraction residues in a fixed-bed reactor: characterization of 
bio-oil and bio-char. J Anal Appl Pyrolys 88:22-29. https://doi. org/10.1016/j.jaap.2010.02.006

Fabbri D et al (2013) Determination of polycyclic aromatic hydrocarbons in biochar and biochar amended soil. J Anal App Pyrolys 103:60-67. https://doi.org/10.1016/j.jaap.2012.10.003

Fawzy S et al (2020) Strategies for mitigation of climate change: a review. Environ Chem Lett 18:2069-2094. https://doi. org/10.1007/s10311-020-01059-w

Ferrer A et al (2016) Analyses of biomass fibers by XRD, FT-IR, and NIR. pp 45-83.https://doi.org/10.1007/978-3-319-41414-0_3

ForestCarbon (2021) https://www.forestcarbon.co.uk. Accessed 27 Jan 2021

Freddo A et al (2012) Environmental contextualisation of potential toxic elements and polycyclic aromatic hydrocarbons in biochar. Environ Pollut 171:18-24. https://doi.org/10.1016/j.envpo 1.2012.07.009

Fuss S et al (2018) Negative emissions-part 2: costs, potentials and side effects. Environ Res Lett 13:063002. https://doi. org/10.1088/1748-9326/aabf9f

Gabhi R et al (2020) Electrical conductivity of wood biochar monoliths and its dependence on pyrolysis temperature. Biochar 2:369-378. https://doi.org/10.1007/s42773-020-00056-0

Gopinath A, Kadirvelu K (2018) Strategies to design modified activated carbon fibers for the decontamination of water and air. Environ Chem Lett 16:1137-1168. https://doi.org/10.1007/s1031 1-018-0740-9

Gopinath KP et al (2021) Environmental applications of carbon-based materials: a review. Environ Chem Lett 19:557-582. https://doi. org/10.1007/s10311-020-01084-9

Gray $M$ et al (2014) Water uptake in biochars: the roles of porosity and hydrophobicity. Biomass Bioenergy 61:196-205. https://doi. org/10.1016/j.biombioe.2013.12.010

Gren I-M, Aklilu AZ (2016) Policy design for forest carbon sequestration: a review of the literature. For Policy Econ 70:128-136. https://doi.org/10.1016/j.forpol.2016.06.008

Gupta S, Kua H (2017) Factors determining the potential of biochar as a carbon capturing and sequestering construction material: critical review. J Mater Civ Eng 29:04017086. https://doi. org/10.1061/(ASCE)MT.1943-5533.0001924

Hale S et al (2012) Quantifying the total and bioavailable polycyclic aromatic hydrocarbons and dioxins in biochars. Environ Sci Technol 46:2830-2838. https://doi.org/10.1021/es203984k

Harvey OR et al (2012) An index-based approach to assessing recalcitrance and soil carbon sequestration potential of engineered black carbons (biochars). Environ Sci Technol 46:1415-1421. https:// doi.org/10.1021/es2040398

Heidari A et al (2014) Effect of process conditions on product yield and composition of fast pyrolysis of Eucalyptus grandis in fluidized bed reactor. J Ind Eng Chem 20:2594-2602. https://doi. org/10.1016/j.jiec.2013.10.046

Heidarinejad Z et al (2020) Methods for preparation and activation of activated carbon: a review. Environ Chem Lett 18:393-415. https ://doi.org/10.1007/s10311-019-00955-0

Henner P., Schiavon M., Morel J.L., Lichtfouse E. (1997) Polycyclic aromatic hydrocarbon (PAH) occurrence and remediation methods. Analusis, 25, M56-M59. https://hal.archives-ouvertes.fr/ hal-00193277

Hirst EA et al (2018) A simple flash carbonization route for conversion of biomass to porous carbons with high $\mathrm{CO}_{2}$ storage capacity. $\mathrm{J}$ Mater Chem A 6:12393-12403. https://doi.org/10.1039/C8TA0 $4409 \mathrm{~K}$

Hornung A (2012) Biomassbiomass pyrolysis biomass pyrolysis. In: Meyers RA (ed) Encyclopedia of sustainability science and technology. Springer, New York, pp 1517-1531. https://doi. org/10.1007/978-1-4419-0851-3_258
Huang Y-F, Lo S-L (2020) Predicting heating value of lignocellulosic biomass based on elemental analysis. Energy 191:116501. https ://doi.org/10.1016/j.energy.2019.116501

IBI (2015) Standardized product definition and product testing guidelines for biochar that is used in soil. Version 2.1. International Biochar Initiative. Accessed 19 Jan 2021. https://www.bioch ar-international.org/wp-content/uploads/2018/04/IBI_Bioch ar_Standards_V2.1_Final.pdf

Ippolito J et al (2020) Feedstock choice, pyrolysis temperature and type influence biochar characteristics: a comprehensive metadata analysis review. Biochar. https://doi.org/10.1007/s4277 3-020-00067-x

Jiang $\mathrm{C}$ et al (2020) Converting waste lignin into nano-biochar as a renewable substitute of carbon black for reinforcing styrenebutadiene rubber. Waste Manag 102:732-742. https://doi. org/10.1016/j.wasman.2019.11.019

Kameyama K et al (2019) The preliminary study of water-retention related properties of biochar produced from various feedstock at different pyrolysis temperatures. Materials 12:1732. https://doi. org/10.3390/ma12111732

Kan T et al (2016) Lignocellulosic biomass pyrolysis: a review of product properties and effects of pyrolysis parameters. Renew Sustain Energy Rev 57:1126-1140. https://doi.org/10.1016/j. rser.2015.12.185

Kazemi Shariat Panahi H et al (2020) A comprehensive review of engineered biochar: production, characteristics, and environmental applications. J Clean Prod 270:122462. https://doi.org/10.1016/j. jclepro.2020.122462

Keiluweit M et al (2012) Solvent-extractable polycyclic aromatic hydrocarbons in biochar: influence of pyrolysis temperature and feedstock. Environ Sci Technol 46:9333-9341. https://doi. org/10.1021/es302125k

Kenney KL et al (2013) Understanding biomass feedstock variability. Biofuels 4:111-127. https://doi.org/10.4155/bfs.12.83

Khushnood RA et al (2016) Carbonized nano/microparticles for enhanced mechanical properties and electromagnetic interference shielding of cementitious materials. Front Struct Civ Eng 10:209-213. https://doi.org/10.1007/s11709-016-0330-5

Kinney TJ et al (2012) Hydrologic properties of biochars produced at different temperatures. Biomass Bioenergy 41:34-43. https://doi. org/10.1016/j.biombioe.2012.01.033

Kloss S et al (2012) Characterization of slow pyrolysis biochars: effects of feedstocks and pyrolysis temperature on biochar properties. J Environ Qual 41:990-1000. https://doi.org/10.2134/jeq20 11.0070

Knicker $\mathrm{H}$ et al (2007) How useful is chemical oxidation with dichromate for the determination of "Black Carbon" in fire-affected soils? Geoderma 142:178-196. https://doi.org/10.1016/j.geode rma.2007.08.010

Kosheleva RI et al (2019) Synthesis of activated carbon from food waste. Environ Chem Lett 17:429-438. https://doi.org/10.1007/ s10311-018-0817-5

Kumar A et al (2020) Advances in design strategies for preparation of biochar based catalytic system for production of high value chemicals. Bioresour Technol 299:122564. https://doi. org/10.1016/j.biortech.2019.122564

Kuppusamy V (2019) Recent advancements in biochar preparation, feedstocks, modification, characterization and future applications. Environ Technol Rev 8:47-64. https://doi. org/10.1080/21622515.2019.1631393

Lawal AT (2017) Polycyclic aromatic hydrocarbons. A review. Cogent Environ Sci 3:1339841. https://doi.org/10.1080/23311 843.2017.1339841

Lehmann J et al (2011) Biochar effects on soil biota-a review. Soil Biol Biochem 43:1812-1836. https://doi.org/10.1016/j.soilb io.2011.04.022 
Leng L, Huang H (2018) An overview of the effect of pyrolysis process parameters on biochar stability. Bioresour Technol 270:627-642. https://doi.org/10.1016/j.biortech.2018.09.030

Leng L et al (2019a) Biochar stability assessment methods: a review. Sci Total Environ 647:210-222. https://doi.org/10.1016/j.scito tenv.2018.07.402

Leng L et al (2019b) Biochar stability assessment by incubation and modelling: methods, drawbacks and recommendations. Sci Total Environ 664:11-23. https://doi.org/10.1016/j.scito tenv.2019.01.298

Li D-C, Jiang H (2017) The thermochemical conversion of non-lignocellulosic biomass to form biochar: a review on characterizations and mechanism elucidation. Bioresour Technol 246:57-68. https ://doi.org/10.1016/j.biortech.2017.07.029

$\mathrm{Li} \mathrm{Y}$ et al (2020) A critical review of the production and advanced utilization of biochar via selective pyrolysis of lignocellulosic biomass. Bioresour Technol 312:123614. https://doi.org/10.1016/j. biortech.2020.123614

Liu B et al (2020) A fast chemical oxidation method for predicting the long-term mineralization of biochar in soils. Sci Total Environ 718:137390. https://doi.org/10.1016/j.scitotenv.2020.137390

Luo L et al (2020) Insight into pyrolysis kinetics of lignocellulosic biomass: isoconversional kinetic analysis by the modified friedman method. Energy Fuels 34:4874-4881. https://doi.org/10.1021/ acs.energyfuels.0c00275

Malghani S et al (2013) Chars produced by slow pyrolysis and hydrothermal carbonization vary in carbon sequestration potential and greenhouse gases emissions. Soil Biol Biochem 62:137-146. https://doi.org/10.1016/j.soilbio.2013.03.013

Man KY et al (2021) Use of biochar as feed supplements for animal farming. Crit Rev Environ Sci Technol 51:187-217. https://doi. org/10.1080/10643389.2020.1721980

Mani T et al (2010) Pyrolysis of wheat straw in a thermogravimetric analyzer: effect of particle size and heating rate on devolatilization and estimation of global kinetics. Chem Eng Res Des 88:952-958. https://doi.org/10.1016/j.cherd.2010.02.008

Manyà JJ et al (2014) Biochar from slow pyrolysis of two-phase olive mill waste: effect of pressure and peak temperature on its potential stability. Energy Fuels 28:3271-3280. https://doi. org/10.1021/ef500654t

Maraseni TN (2010) Biochar: maximising the benefits. Int J Environ Stud 67:319-327. https://doi.org/10.1080/00207231003612225

Matamba $\mathrm{T}$ et al (2020) Promotion effects of pressure on polycyclic aromatic hydrocarbons and $\mathrm{H}_{2}$ formation during flash pyrolysis of palm kernel shell. Energy Fuels 34:3346-3356. https://doi. org/10.1021/acs.energyfuels.9b04409

Melligan F et al (2011) Pressurised pyrolysis of Miscanthus using a fixed bed reactor. Bioresour Technol 102:3466-3470. https://doi. org/10.1016/j.biortech.2010.10.129

Meyer-Kohlstock D et al (2016) Biochar as Additive in biogas-production from bio-waste. Energies 9:247. https://doi.org/10.3390/ en9040247

Mia S et al (2017) Aged biochar affects gross nitrogen mineralization and recovery: a $15 \mathrm{~N}$ study in two contrasting soils. GCB Bioenergy 9:1196-1206. https://doi.org/10.1111/gcbb.12430

Mishra RK, Mohanty K (2018) Pyrolysis kinetics and thermal behavior of waste sawdust biomass using thermogravimetric analysis. Bioresour Technol 251:63-74. https://doi.org/10.1016/j.biort ech.2017.12.029

Mok WSL, Antal MJ (1983) Effects of pressure on biomass pyrolysis. II. Heats of reaction of cellulose pyrolysis. Thermochimica Acta 68:165-186. https://doi.org/10.1016/0040-6031(83)80222-6

Mudhoo A et al (2019) Green synthesis, activation and functionalization of adsorbents for dye sequestration. Environ Chem Lett 17:157-193. https://doi.org/10.1007/s10311-018-0784-x
Mulabagal V et al (2017) Biochar from biomass: a strategy for carbon dioxide sequestration, soil amendment, power generation, and $\mathrm{CO}_{2}$ utilization. In: Chen W-Y et al (eds) Handbook of climate change mitigation and adaptation. Springer, Cham, pp 19371974. https://doi.org/10.1007/978-3-319-14409-2_80

Munera-Echeverri JL et al (2018) Cation exchange capacity of biochar: an urgent method modification. Sci Total Environ 642:190-197. https://doi.org/10.1016/j.scitotenv.2018.06.017

Nartey OD, Zhao B (2014) Biochar preparation, characterization, and adsorptive capacity and its effect on bioavailability of contaminants: an overview. Adv Mater Sci Eng 2014:715398. https://doi. org/10.1155/2014/715398

Nori (2021) https://nori.com. Accessed 27 Jan 2021

Novotny EH et al (2015) Biochar: pyrogenic carbon for agricultural use-a critical review. Revista Brasileira de Ciência do Solo 39:321-344

Nunoura $\mathrm{T}$ et al (2006) Studies of the flash carbonization process. 1. Propagation of the flaming pyrolysis reaction and performance of a catalytic afterburner. Ind Eng Chem Res 45:585-599. https ://doi.org/10.1021/ie050854y

Okafor D, Daramola MO (2020) A short overview of analytical techniques in biomass feedstock characterization, pp 21-46. https:// doi.org/10.1007/978-3-030-38032-8_2

Onay O (2007) Influence of pyrolysis temperature and heating rate on the production of bio-oil and char from safflower seed by pyrolysis, using a well-swept fixed-bed reactor. Fuel Process Technol 88:523-531. https://doi.org/10.1016/j.fuproc.2007.01.001

OneTreePlanted (2021) https://onetreeplanted.org. Accessed 27 Jan 2021

Oni BA et al (2019) Significance of biochar application to the environment and economy. Ann Agric Sci 64:222-236. https://doi. org/10.1016/j.aoas.2019.12.006

Osman AI et al (2018) Physicochemical characterization of miscanthus and its application in heavy metals removal from wastewaters. Environ Prog Sustain Energy 37:1058-1067. https://doi. org/10.1002/ep.12783

Osman AI et al (2019) Production and characterisation of activated carbon and carbon nanotubes from potato peel waste and their application in heavy metal removal. Environ Sci Pollut Res 26:37228-37241. https://doi.org/10.1007/s11356-019-06594-w

Osman AI et al (2020a) Recent advances in carbon capture storage and utilisation technologies: a review. Environ Chem Lett. https://doi. org/10.1007/s10311-020-01133-3

Osman AI et al (2020b) Physicochemical characterization and kinetic modeling concerning combustion of waste berry pomace. ACS Sustain Chem Eng. https://doi.org/10.1021/acssuschem eng.0c07390

Osman AI et al (2020c) Pyrolysis kinetic modelling of abundant plastic waste (PET) and in-situ emission monitoring. Environ Sci Europe 32:112. https://doi.org/10.1186/s12302-020-00390-x

Ozbay N et al (2006) Bio-oil production from rapid pyrolysis of cottonseed cake: product yields and compositions. Int J Energy Res 30:501-510. https://doi.org/10.1002/er.1165

Purakayastha TJ et al (2019) A review on biochar modulated soil condition improvements and nutrient dynamics concerning crop yields: pathways to climate change mitigation and global food security. Chemosphere 227:345-365. https://doi.org/10.1016/j. chemosphere.2019.03.170

Puro.earth (2021a) https://puro.earth. Accessed 27 Jan 2021

Puro.earth (2021b) Puro.earth $\mathrm{CO}_{2}$ removal marketplace: general rules. Version 2.0 https://static.puro.earth/live/uploads/tinymce/Puro_ Documents/Puro-Rules-CO2-removal-marketplace_v2.0_final .pdf. Accessd: 29 Jan 2021

Qambrani NA et al (2017) Biochar properties and eco-friendly applications for climate change mitigation, waste management, and 
wastewater treatment: a review. Renew Sustain Energy Rev 79:255-273. https://doi.org/10.1016/j.rser.2017.05.057

$\mathrm{Ra} A$ et al (2016) Biochar applications and modern techniques for characterization. Clean Technol Environ Policy. https://doi. org/10.1007/s10098-016-1218-8

Rajkovich S et al (2011) Corn growth and nitrogen nutrition after additions of biochars with varying properties to a temperate soil. Biol Fertil Soils. https://doi.org/10.1007/s00374-011-0624-7

Rayment G, Higginson F (1992) Australian laboratory handbook of soil and water chemical methods

Restuccia L, Ferro G (2016) Nanoparticles from food waste: a "green" future for traditional building materials. https://doi.org/10.21012 /FC9.276

Richard J-R, Antal MJ (1993) Thermogravimetric Studies of charcoal formation from cellulose at elevated pressures. In: Bridgwater AV (ed) Advances in thermochemical biomass conversion. Springer, Dordrecht, pp 784-792.https://doi. org/10.1007/978-94-011-1336-6_61

Rogovska N et al (2012) Germination tests for assessing biochar quality. J Environ Qual 41:1014-1022. https://doi.org/10.2134/jeq20 11.0103

Salehi E et al (2009) Bio-oil from sawdust: pyrolysis of sawdust in a fixed-bed system. Energy Fuels 23:3767-3772. https://doi. org/10.1021/ef900112b

Schmidt H-P (2013) 55 Uses of Biochar. Ithaka J

Schmidt H-P et al (2019) Pyrogenic carbon capture and storage. GCB Bioenergy 11:573-591. https://doi.org/10.1111/gcbb.12553

Semida WM et al (2019) Biochar implications for sustainable agriculture and environment: a review. S Afr JBot 127:333-347. https ://doi.org/10.1016/j.sajb.2019.11.015

Şensöz S, Angın D (2008) Pyrolysis of safflower (Charthamus tinctorius L.) seed press cake: Part 1 . The effects of pyrolysis parameters on the product yields. Bioresour Technol 99:5492-5497. https://doi.org/10.1016/j.biortech.2007.10.046

Shabangu S et al (2014) Techno-economic assessment of biomass slow pyrolysis into different biochar and methanol concepts. Fuel 117:742-748. https://doi.org/10.1016/j.fuel.2013.08.053

Sharma $R$ et al (2019) A comprehensive review on hydrothermal carbonization of biomass and its applications

Singh B et al (2017) Chapter 3. Biochar $\mathrm{pH}$, electrical conductivity and liming potential, pp 23-38

Spokas KA (2010) Review of the stability of biochar in soils: predictability of O:C molar ratios. Carbon Manag 1:289-303. https://doi. org $/ 10.4155 / \mathrm{cmt} .10 .32$

Tenic E et al (2020) Biochar-a panacea for agriculture or just carbon? Horticulturae. https://doi.org/10.3390/horticulturae6030037

Tisserant A, Cherubini F (2019) Potentials, limitations, co-benefits, and trade-offs of biochar applications to soils for climate change mitigation. Land 8:179. https://doi.org/10.3390/land8120179

Tomczyk A et al (2020) Biochar physicochemical properties: pyrolysis temperature and feedstock kind effects. Rev Environ Sci Bio/ Technol 19:191-215. https://doi.org/10.1007/s11157-020-09523-3

Tripathi M et al (2016) Effect of process parameters on production of biochar from biomass waste through pyrolysis: a review. Renew Sustain Energy Rev 55:467-481. https://doi.org/10.1016/j. rser.2015.10.122

Uddin MN et al (2018) An overview of recent developments in biomass pyrolysis technologies. Energies. https://doi.org/10.3390/en111 13115

Ulusal A et al (2020) Opportunity for sustainable biomass valorization to produce biochar for improving soil characteristics. Biomass Convers Biorefinery. https://doi.org/10.1007/s13399-020-00923-7

Usevičiūte L, Baltrènaitė E (2020) Methods for determining lignocellulosic biochar wettability. Waste Biomass Valoriz 11:4457-4468. https://doi.org/10.1007/s12649-019-00713-x
Usevičiūtė L, Baltrėnaitė-Gedienė E (2020) Dependence of pyrolysis temperature and lignocellulosic physical-chemical properties of biochar on its wettability. Biomass Convers Biorefinery. https:// doi.org/10.1007/s13399-020-00711-3

Van Zwieten L et al (2010) Effects of biochar from slow pyrolysis of papermill waste on agronomic performance and soil fertility. Plant Soil 327:235-246. https://doi.org/10.1007/s11104-009-0050-x

Várhegyi G et al (1998) TG, TG-MS, and FTIR characterization of highyield biomass charcoals. Energy Fuels 12:969-974. https://doi. org/10.1021/ef9800359

Vyazovkin S et al (2011) ICTAC Kinetics Committee recommendations for performing kinetic computations on thermal analysis data. Thermochimica Acta 520:1-19. https://doi.org/10.1016/j. tca.2011.03.034

Waluyo J et al (2018) Pyrolysis with intermediate heating rate of palm kernel shells: effect temperature and catalyst on product distribution. AIP Conf Proc 1977:020026. https://doi.org/10.1063/1.50428 82

Wambugu CW et al (2019) Role of biochar in anaerobic digestion based biorefinery for food waste. Front Energy Res. https://doi. org/10.3389/fenrg.2019.00014

Wang T et al (2012) Predicting phosphorus bioavailability from high-ash biochars. Plant Soil. https://doi.org/10.1007/s11104-012-1131-9

Wang J et al (2016) Biochar stability in soil: meta-analysis of decomposition and priming effects. GCB Bioenergy 8:512-523. https://doi. org/10.1111/gcbb.12266

Wang C et al (2017) Polycyclic aromatic hydrocarbons (PAHs) in biochar-their formation, occurrence and analysis: a review. Organ Geochem 114:1-11. https://doi.org/10.1016/j.orggeochem .2017.09.001

Wang B et al (2018) Recent advances in engineered biochar productions and applications. Crit Rev Environ Sci Technol 47:1-50. https:// doi.org/10.1080/10643389.2017.1418580

Wang D et al (2020) Biochar production and applications in agro and forestry systems: a review. Sci Total Environ 723:137775. https:// doi.org/10.1016/j.scitotenv.2020.137775

Wiedemeier DB et al (2015) Aromaticity and degree of aromatic condensation of char. Organ Geochem 78:135-143. https://doi. org/10.1016/j.orggeochem.2014.10.002

Williams PT, Nugranad N (2000) Comparison of products from the pyrolysis and catalytic pyrolysis of rice husks. Energy 25:493-513. https ://doi.org/10.1016/S0360-5442(00)00009-8

WoodlandTrust (2021) https://www.woodlandtrust.org.uk. Accessed 27 Jan 2021

Wu B et al (2020) Methane emission reduction enhanced by hydrophobic biochar-modified soil cover. Processes. https://doi.org/10.3390/ pr8020162

Xiao L et al (2019) Biochar promotes methane production at high acetate concentrations in anaerobic soils. Environ Chem Lett 17:13471352. https://doi.org/10.1007/s10311-019-00863-3

$\mathrm{Xu} \mathrm{F}$ et al (2013) Qualitative and quantitative analysis of lignocellulosic biomass using infrared techniques: a mini-review. Appl Energy 104:801-809. https://doi.org/10.1016/j.apenergy.2012.12.019

$\mathrm{Xu} \mathrm{M}$ et al (2019) Biochar addition to soil highly increases P retention and decreases the risk of phosphate contamination of waters. Environ Chem Lett 17:533-541. https://doi.org/10.1007/s1031 1-018-0802-z

Yaashikaa PR et al (2019) Advances in production and application of biochar from lignocellulosic feedstocks for remediation of environmental pollutants. Bioresour Technol. 292:122030. https://doi. org/10.1016/j.biortech.2019.122030

Yang Y et al (2014) Intermediate pyrolysis of biomass energy pellets for producing sustainable liquid, gaseous and solid fuels. Bioresour Technol 169:794-799. https://doi.org/10.1016/j.biort ech.2014.07.044 
Yargicoglu EN et al (2015) Physical and chemical characterization of waste wood derived biochars. Waste Manag 36:256-268. https:// doi.org/10.1016/j.wasman.2014.10.029

Zeng $\mathrm{K}$ et al (2015) The effect of temperature and heating rate on char properties obtained from solar pyrolysis of beech wood. Bioresour Technol 182:114-119. https://doi.org/10.1016/j.biort ech.2015.01.112

Zhang $\mathrm{H}$ et al (2009) Comparison of non-catalytic and catalytic fast pyrolysis of corncob in a fluidized bed reactor. Bioresour Technol 100:1428-1434. https://doi.org/10.1016/j.biortech.2008.08.031

Zhang $M$ et al (2020) Evaluating biochar and its modifications for the removal of ammonium, nitrate, and phosphate in water. Water Res 186:116303. https://doi.org/10.1016/j.watres.2020.116303

Zhao $\mathrm{S}$ et al (2014a) Laboratory investigation of bio-char modified asphalt mixtures. Transp Res Rec J Transp Res Board. https://doi. org/10.3141/2445-07
Zhao $\mathrm{S}$ et al (2014b) Utilizing bio-char as a bio-modifier for asphalt cement: a sustainable application of bio-fuel by-product. Fuel 133:52-62. https://doi.org/10.1016/j.fuel.2014.05.002

Zhou $\mathrm{H}$ et al (2014) Effect of interactions of biomass constituents on polycyclic aromatic hydrocarbons (PAH) formation during fast pyrolysis. J Anal Appl Pyrolys 110:264-269. https://doi.org/10.1016/j. jaap.2014.09.007

Zielińska A, Oleszczuk P (2015) The conversion of sewage sludge into biochar reduces polycyclic aromatic hydrocarbon content and ecotoxicity but increases trace metal content. Biomass Bioenergy 75:235-244. https://doi.org/10.1016/j.biombioe.2015.02.019

Publisher's Note Springer Nature remains neutral with regard to jurisdictional claims in published maps and institutional affiliations. 